\title{
Direct and indirect effects of multilingualism on novel language learning: An integrative review
}

\author{
Zoya Hirosh $^{1} \cdot$ Tamar Degani $^{1}$
}

Published online: 25 May 2017

(C) Psychonomic Society, Inc. 2017

\begin{abstract}
Accumulated recent research suggests that prior knowledge of multiple languages leads to advantages in learning additional languages. In the current article, we review studies examining potential differences between monolingual and multilingual speakers in novel language learning in an effort to uncover the cognitive mechanisms that underlie such differences. We examine the multilingual advantage in children and adults, across a wide array of languages and learner populations. The majority of this literature focused on vocabulary learning, but studies that address phonology, grammar, and literacy learning are also discussed to provide a comprehensive picture of the way in which multilingualism affects novel language learning. Our synthesis indicates two avenues to the multilingual advantage including direct transfer of prior knowledge and prior skills as well as indirect influences that result from multilingual background and include more general changes to the cognitive-linguistic system. Finally, we highlight topics that are in need of future systematic research.
\end{abstract}

Keywords Multilingualism $\cdot$ Bilingualism $\cdot$ Novel language learning $\cdot$ Transfer $\cdot$ Vocabulary $\cdot$ Phonology $\cdot$ Grammar · Literacy

Recent research suggests that multilinguals may differ from monolinguals in language learning. When learning a novel language, multilinguals are typically better than monolinguals in different linguistic aspects of the novel language, including

Tamar Degani

tdegani@ research.haifa.ac.il

1 Department of Communication Sciences \& Disorders, University of Haifa, Mount Carmel, Haifa, Israel vocabulary (Kalashnikova, Mattock, \& Monaghan, 2014; Kaushanskaya \& Marian, 2009a, 2009b), phonology (e.g., Antoniou, Liang, Ettlinger, \& Wong, 2015; Wang \& Saffran, 2014), grammar (Klein, 1995; Sanz, 2000, 2007), and literacy (e.g., Kahn-Horwitz, Kuash, Ibrahim, \& Schwartz, 2014). The present article integrates and reviews these recent findings to identify the cognitive mechanism(s) that may underlie such an advantage. To date, different explanations have been suggested to account for the multilingual advantage, including extensive experience with mapping novel labels to known concepts (Kaushanskaya, 2012), experience with competing mappings of two phonological systems onto the same orthography (Kaushanskaya \& Marian, 2009a), reliance on meaning (Kaushanskaya \& Rechtzigel, 2012), enhanced cognitive control abilities (Bartolotti \& Marian, 2012; Bartolotti, Marian, Schroeder \& Shook, 2011; Bogulski, Bice, \& Kroll (in preparation), 2013; Kaushanskaya \& Marian, 2009b; Wang \& Saffran, 2014), and enhanced metalinguistic awareness (Modirkhamene, 2006; Sanz, 2000).

To highlight the possible contribution of these and additional explanations, we discuss the findings with reference to the general manner in which multilingualism may affect language learning. Specifically, as detailed in Fig. 1, we propose that the multilingual effect on language learning includes both direct and indirect influences, which affect different aspects of language learning including knowledge (vocabulary learning, phonology, grammar, and literacy) as well as fluency in the novel language. Direct effects are those that transfer "as is" from earlier experiences to the task at hand. These processes include both transfer of knowledge and representations from known languages as well as implementation of practiced learning strategies and skills. Thus, both established representations as well as practiced processes can facilitate current learning. These direct influences critically depend on the degree of similarity between the known languages and the to-be- 


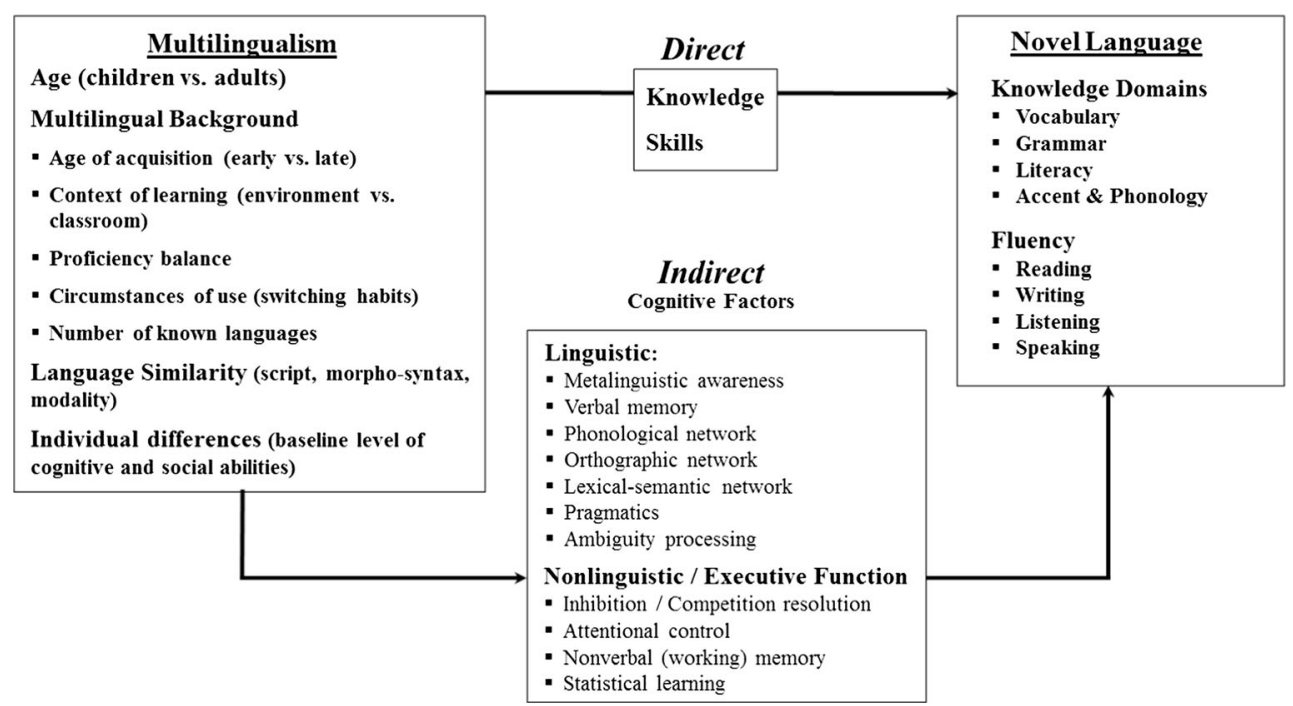

Fig. 1 Theoretical framework for the influence of multilingualism on novel language learning

learned language, as well as between prior learning and processing experiences and the current learning task.

Notably, the framework we propose emphasizes that multilingualism may further exert an indirect effect, which reflects some involvement of additional mediating factors that were influenced by prior experiences. For instance, prior experiences may have led to changes in the cognitive and social abilities of the learner. When faced with novel language learning, these enhanced abilities facilitate learning. The cognitive and social abilities are considered mediators in the indirect pathway by which multilingualism enhances novel language learning. These interceding abilities could be described as linguistic or nonlinguistic in nature but critically work indirectly to enhance multilinguals' language learning.

As detailed in the figure and throughout the article, all of these effects should be examined in the context of the particular characteristics of the learners and the languages in question. Speakers of different ages (adults vs. children), with different L2 age of acquisition and or proficiency, different L2 learning circumstances, and different language pairs (overlapping to various degrees), may differentially utilize their direct and indirect resources when facing novel language learning. Indeed, multilingualism is not a uniform phenomenon (Kroll \& Bialystok, 2013; Kaushanskaya \& Prior, 2015), such that all of the above factors shape the multilingual profile. Similarly, the number of languages known by the multilingual speaker is critical to consider. Thus, bilinguals may differ from trilinguals or from speakers of even more languages in the resources they bring to the learning situation (e.g., Herdina \& Jessner, 2002; Jessner, 2008). However, we view this as one dimension on which multilinguals vary. Thus, in the literature that we review, we consider comparisons between monolinguals and speakers of more than one language as the defining criterion for inclusion in the review. Studies that report on differences between monolinguals and bilinguals, or between monolinguals and trilinguals or other multilinguals, are all relevant when examining the multilingual effect on novel language learning because bilinguals and other multilinguals all have enhanced experience with language learning compared to monolinguals. We return to this issue in the General Framework section.

We begin our review by comparing the performance of monolinguals versus multilinguals on different aspects of language learning, including word learning, phonology, grammar, and literacy. We then discuss these findings with reference to the general framework of direct and indirect effects and consider how these effects are modulated by learner and language characteristics. We conclude with issues that call for future systematic research. Table 1 presents a summary of the reviewed literature highlighting relevant characteristics of the studies.

\section{Different aspects of language learning}

\section{Word learning}

A growing body of literature examines differences between multilinguals and monolinguals in novel word learning. This literature suggests advantages for multilinguals over monolinguals both among adults (e.g., Bartolotti \& Marian, 2012; Bogulski et al., in preparation; Kaushanskaya, 2012; Kaushanskaya \& Marian, 2009a, 2009b; Kaushanskaya \& Rechtzigel, 2012; Kaushanskaya, Yoo, \& van Hecke, 2013; Keshavarz \& Astaneh, 2004; Papagano \& Vallar 1995; Wang \& Saffran, 2014; Van Hell \& Candia Mahn, 1997) and among children (e.g., Brojde, Ahmed, \& Colunga, 2012; Kalashnikova et al., 2014; Kaushanskaya, Gross, \& Buac, 2014; Yoshida, Tran, Benitez, \& Kuwabara, 2011). 


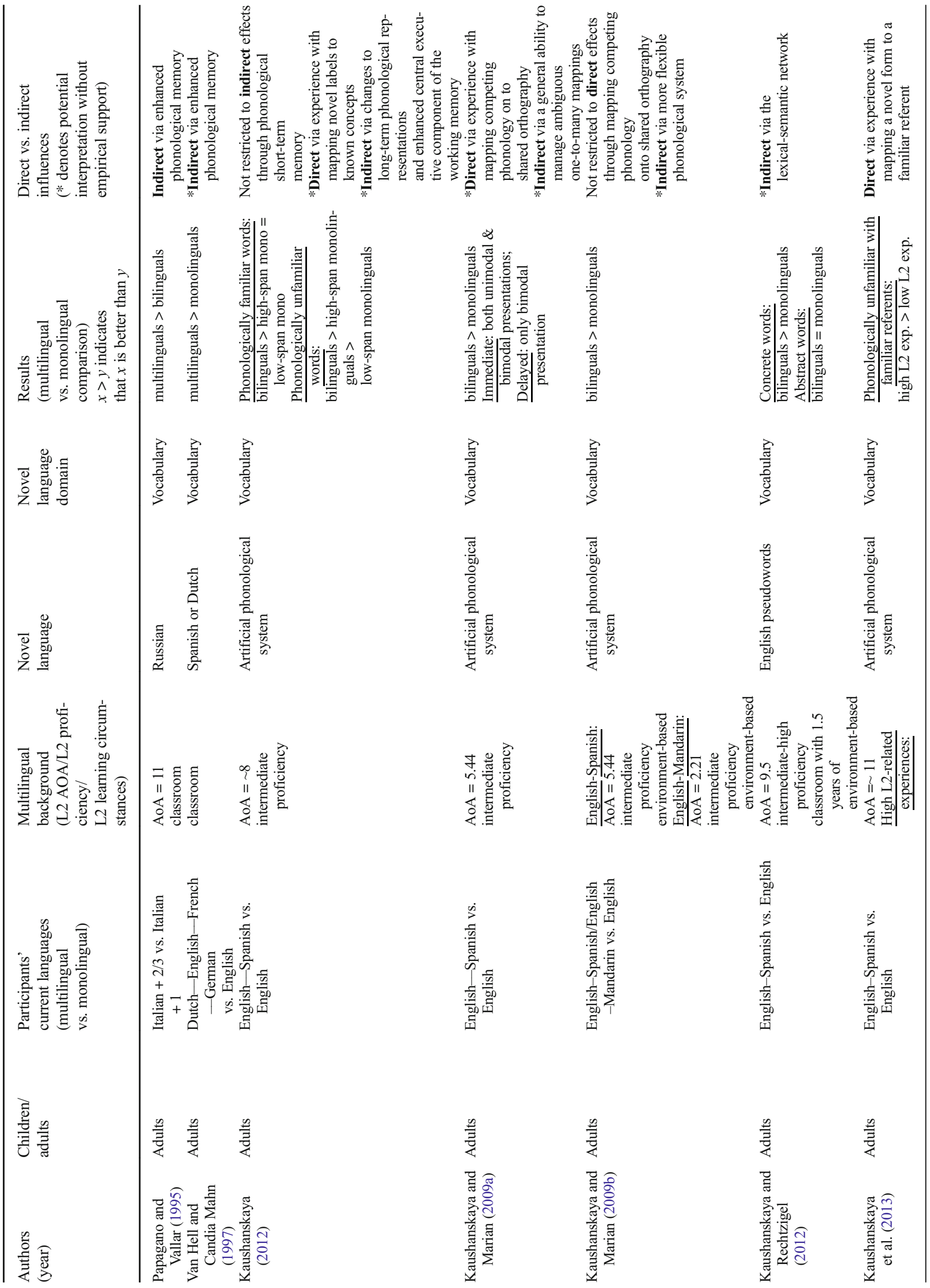




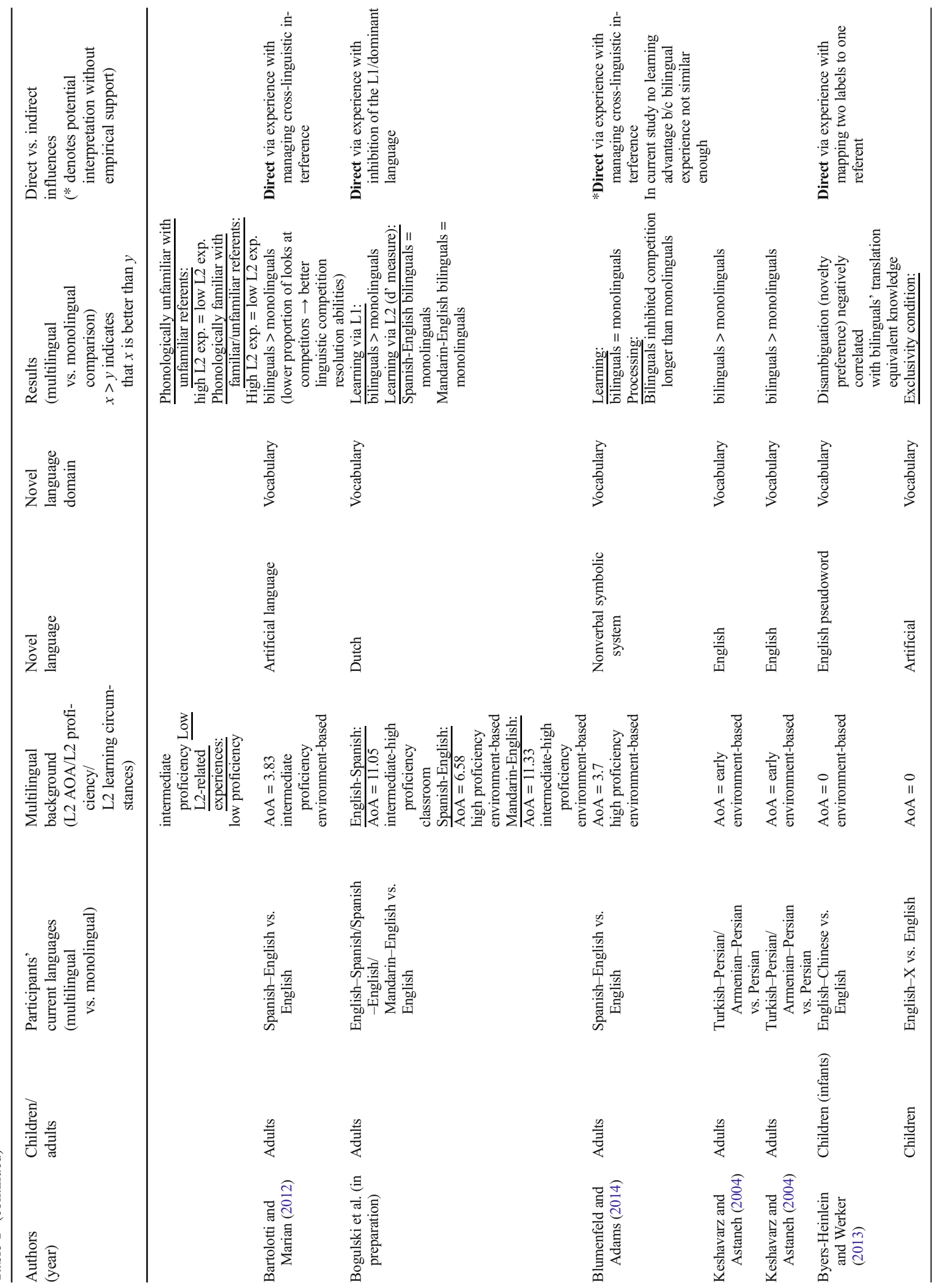




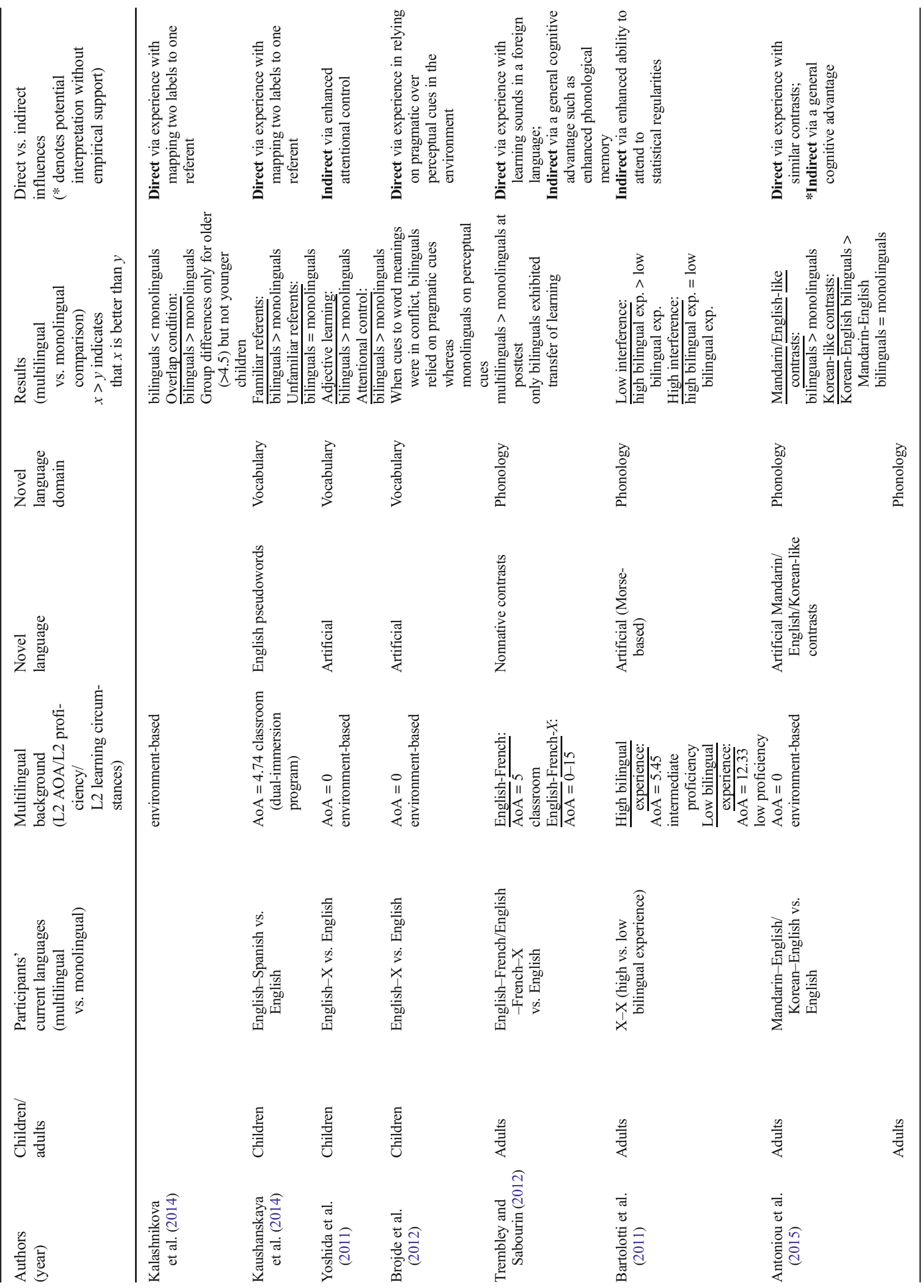




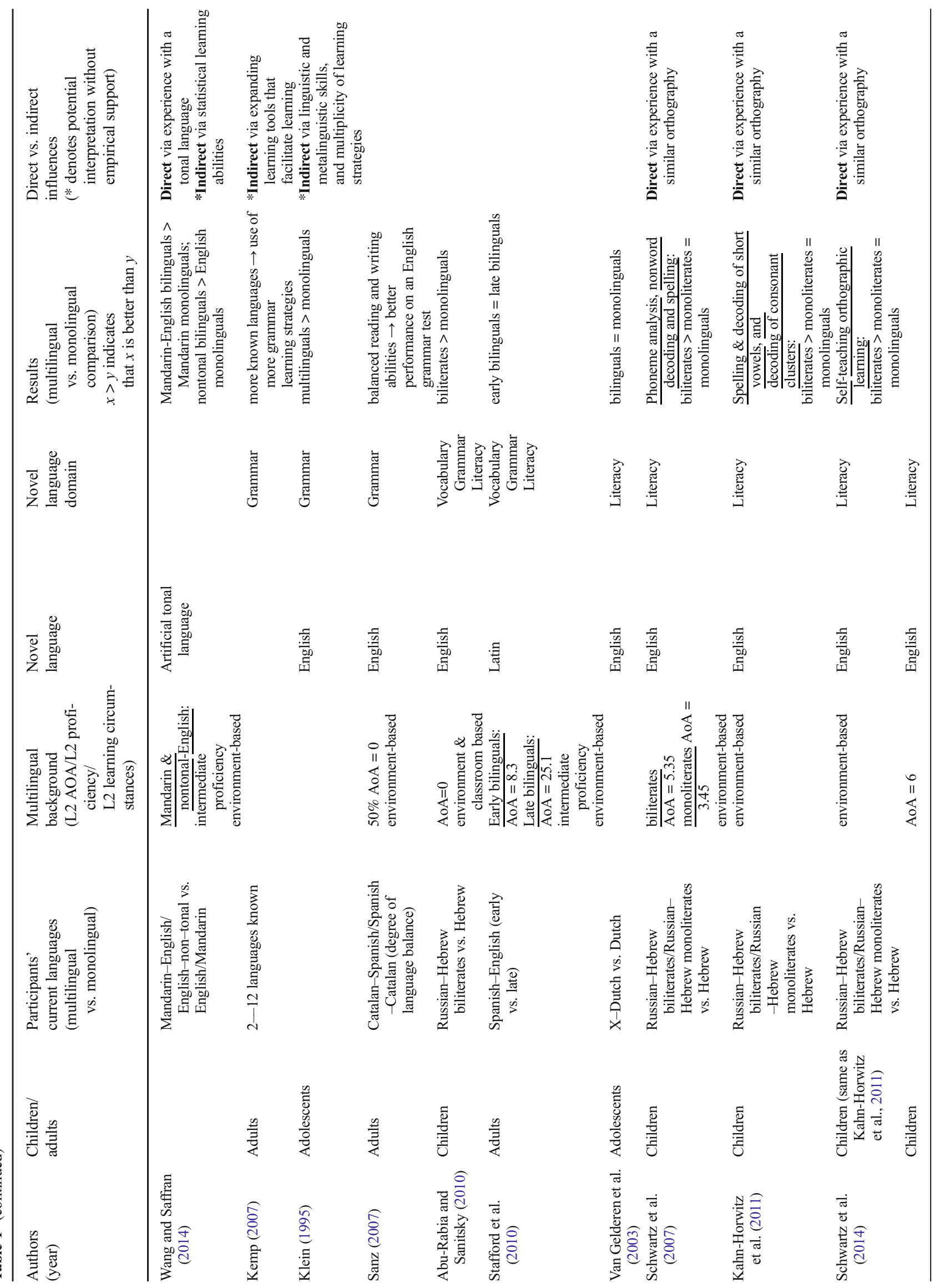




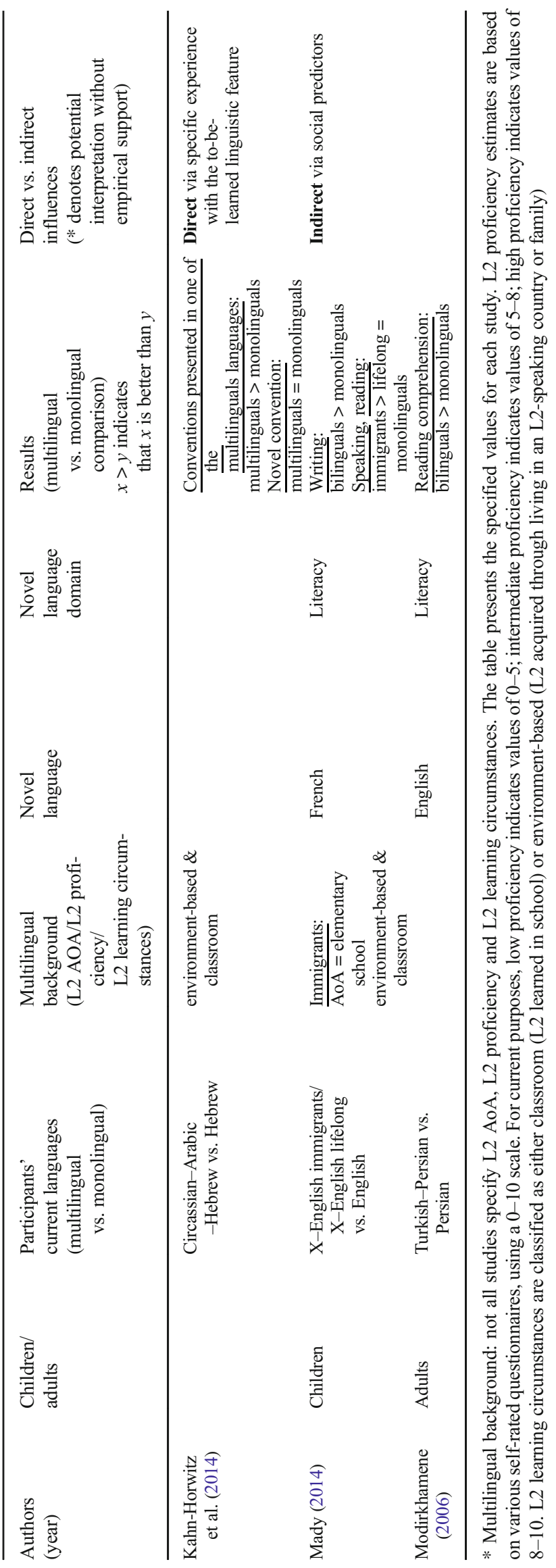




\section{Adults}

Suggestive evidence for the effect of language learning experience on word learning comes from an early study, which compared multilinguals to bilinguals (Papagano \& Vallar, 1995). They compared adult multilinguals (speakers of Italian and two or three other languages) to bilingual speakers of Italian and only one other language on two learning tasks. Specifically in the novel word-learning task, Russian words (transliterated into Italian) were paired with the corresponding Italian translations. In a second task, two Italian words were paired with each other, such that participants were to learn the association between two known words. The results showed significantly higher recall rates for multilinguals on the wordlearning task, but not on the paired associate learning. The authors attributed the learning advantage to superior phonological memory of multilinguals for two reasons. First, they observed significantly better nonword repetition and digit span performance for multilinguals over bilinguals. Second, participants' phonological memory as measured by these tasks was significantly positively correlated with participant's novel word learning (i.e., of the Russian nonnative words). Within the framework we propose, such a suggestion is consistent with an indirect effect of multilingualism on language learning operating through the phonological memory system.

Van Hell and Candia Mahn (1997) compared multilinguals (native Dutch speakers who had learned English, French, and German in a school context) to monolinguals (native English speakers) in learning novel vocabulary (Spanish for multilinguals and Dutch for monolinguals). Across two teaching methods (keyword and rote rehearsal), they observed a learning advantage for the more experienced language learners. They attributed the multilingual advantage to superior phonological memory, but the mechanism was not empirically examined.

To specifically test the role of the phonological memory system in the multilingual word learning advantage, Kaushanskaya (2012) used an artificial phonological system and compared learning of words consisting of familiar phonemes only to words consisting of familiar and unfamiliar phonemes. English-Spanish bilinguals were compared to two groups of English monolinguals: those that matched bilinguals on phonological memory performance (high-span monolinguals) and those that scored lower on the phonological memory tests (low-span monolinguals). Results showed that bilinguals outperformed both monolingual groups when learning both the phonologically familiar and unfamiliar novel words. However, high-span monolinguals outperformed lowspan monolinguals only on the phonologically unfamiliar words. Based on these patterns, Kaushanskaya suggested that phonological memory capacity is more predictive of word learning when the novel words are less familiar. Indeed, Papagano and Vallar (1995) observed a difference between multilinguals (with higher phonological memory) over bilinguals (with lower phonological memory) only in learning phonologically unfamiliar (Russian) words but not in the pairedassociate task.

As was noted by Kaushanskaya (2012), the above studies examined multilinguals that have had extensive classroombased exposure to their multiple languages. Such multilinguals not only know multiple languages but have also gained extensive experience in learning novel vocabulary in formal settings. The situation is different for multilinguals who acquired their languages through the environment, because although they, too, know multiple languages, they likely had little explicit experience with vocabulary learning. These environment-based ${ }^{1}$ multilinguals include immersion-based multilinguals as well as lifelong multilinguals, both of which do not substantially differ from monolinguals in the experience they have accumulated in classroom-based vocabulary learning throughout their lives. Thus, to be able to discern whether these multilingual advantages result from the knowledge of multiple languages per se, or whether they stem from specific prior experience with language learning, environment-based multilinguals should be examined.

In a series of studies, Kaushanskaya and Marian (2009a, 2009b) demonstrated that even environment-based multilinguals, who acquired their languages through unstructured contexts, outperformed monolinguals in novel word learning and that this difference is again not rooted in phonological shortterm memory. Specifically, Kaushanskaya and Marian (2009a) showed that English-Spanish bilingual adults, who acquired their two languages in unstructured contexts early in life, outperformed English monolinguals on a wordlearning task where artificially constructed phonologically unfamiliar novel words were paired with native-language English translations. The words were learned either unimodally (hearing the novel word and seeing its English translation) or bimodally (hearing the novel word and seeing its written form in Latin alphabet along with the English translation). Although the two groups did not differ in phonological short-term memory capacity, bilinguals outperformed monolinguals in both unimodal and bimodal learning tasks immediately after learning. After a delay, the bilingual advantage was only significant in the bimodal presentation condition. Because the bimodal condition involves mapping unfamiliar phonology onto a familiar orthography (i.e., the Latin alphabet), Kaushanskaya and Marian suggested that prior exposure to two languages, that involve competition between

\footnotetext{
${ }^{1}$ We introduce the term environment-based multilinguals to refer to multilinguals who learn their languages through unstructured contexts. These are contrasted with classroom-based multilinguals who learn their languages in formal, structured, situations. We consider both lifelong (simultaneous) multilinguals and immersion-based learners (or immigrants) as environment-based multilinguals because the majority of their learning is based on unstructured encounters with the language, although clearly the two groups differ in AoA.
} 
phonology and orthography, may reduce interference in subsequent learning situations. Specifically, experience with Spanish that matches English in orthography but not in phonology reduces future interference from the strong (L1) letterto-phoneme mappings while learning new linguistic information, and thus facilitate bilinguals' novel language learning.

Within the framework we propose, prior experience with mapping competing phonology on to shared orthography may be thought of as a specific skill that is trained by prior experience, leading to direct implementation of this skill when future language learning requires similar mappings of two phonologies on to one orthography. Alternatively, such experience may work to enhance multilinguals' general ability to deal with ambiguous mappings during learning, not just between phonology and orthography but also between other kinds of representations (e.g., phonology and meaning). Thus, the speaker may develop a general ability to entertain ambiguous one-to-many mappings within the linguistic system, and this ability is then utilized when learning a foreign language. When viewed in this way, experience with mapping two phonologies onto one orthography strengthened ambiguity processing as a general linguistic ability, and this ability works indirectly as a mediating factor facilitating word learning. If this is the case, then multilinguals may be better not only when mapping two phonologies onto a single orthography but also when dealing with ambiguity more generallyfor instance, when processing mappings of two meanings onto a shared form (i.e., semantic ambiguity within and across languages; Degani \& Tokowicz, 2010), mapping two forms onto a shared meaning (i.e., synonymy), etc. We return to this suggestion in the General Framework section.

Interestingly, it appears that the multilingual word learning advantage is not dependent on direct implementation of practiced many-to-one mapping skills of phonology to orthography. First, multilinguals outperformed monolinguals even in the unimodal condition (immediately after learning), when there is no need to map competing phonologies onto a shared orthography (Kaushanskaya \& Marian, 2009a). Further, in a second study, Kaushanskaya and Marian (2009b) showed that early English-Spanish and early English-Mandarin bilinguals similarly outperformed English monolinguals on a wordlearning task using the same artificial phonological system (although the three groups did not differ in phonological short-term memory). These results show that prior experience with mapping competing phonologies onto a single orthographic system is not a requirement because English and Mandarin have nonoverlapping orthographic systems. Instead, the authors proposed that the critical dimension is early experience with two phonological systems. Exposure to such rich and varied speech input may lead to a more flexible phonological system, which allows for efficient encoding of unfamiliar phonological information. Within the framework we propose, this explanation may be seen as indirect in nature, influencing multilingual language learning via changes to the phonological system as a whole, making it more malleable and open to change. Prior word learning leads to changes in the phonological system, which in turn allows for better novel vocabulary learning.

Relatedly, the multilingual advantage for both SpanishEnglish and Mandarin-English bilinguals may be rooted in the speculated enhanced ambiguity processing suggested above. Specifically, we may hypothesize that multilinguals accumulated increased experience with mapping two labels (translations) onto a shared concept, compared to monolinguals. This experience may have led to enhanced ability to manage indirect mappings, which in turn promotes novel language learning. If prior experience involved indirect mappings between two labels and one meaning, and this same type of indirect mapping is required during the novel learning task, then the mechanism can be viewed as direct, because practiced skills are being transferred as is to the novel learning situation. At the same time, if one type of ambiguity management was practiced in the past (e.g., mapping two phonologies onto one meaning) but a different type of ambiguity is now required during novel learning (e.g., mapping two phonologies onto one orthography), then this mechanism should be viewed as an indirect source for the multilingual advantage.

The studies described thus far have demonstrated a multilingual advantage in novel word learning that is not restricted to classroom-based L2 learners and that is not dependent on enhanced phonological short-term memory capacity or experience with mapping competing phonologies onto a shared orthography. An additional source of the multilingual advantage in word learning has been suggested by Kaushanskaya and Rechtzigel (2012), who examined whether multilinguals are more sensitive to semantic information associated with the presented novel words. They compared adult EnglishSpanish bilinguals (with at least some immersion-based experience) to English monolinguals, contrasting learning of novel words in association with concrete versus abstract English translations. The results showed a multilingual advantage when novel words were associated with concrete concepts but not when novel words were associated with abstract concepts. Moreover, although both bilingual and monolingual participants were more accurate in learning concrete rather than abstract words, the concreteness effect was stronger for the bilingual group. The authors suggested that the multilingual advantage in word learning is most likely to emerge when the semantic information associated with the novel word is accessible. Concrete-translation pairs have been suggested to enjoy a larger semantic overlap across languages than do abstract translation pairs (e.g., De Groot, 1992; Van Hell \& De Groot, 1998). Therefore, whereas concrete words cause activation of both of a bilinguals' lexical-semantic networks (i.e., in both English and Spanish), abstract words are likely to lead to more restricted monolingual-type activation in the lexical- 
semantic system. The wider activation of the lexical-semantic system yields better learning of concrete words in bilinguals than in monolinguals. Thus, Kaushanskaya and Rechtzigel (2012) suggest that the multilingual advantage in word learning may be rooted in the organization and activation pattern of the lexical-semantic network. Such a proposal fits in with our suggestion of indirect effects, by which the multilingual experience influences the lexical-semantic network, which in turn facilitates certain novel vocabulary learning. Extending this suggestion further, to the extent that multilinguals differ from monolinguals in the organization of their lexicalsemantic network, we would predict differences between multilinguals and monolinguals in other semantically based outcome measures, such as semantic decisions on word pairs and prediction during sentence reading, and this novel proposal awaits further investigation.

In a related study, Kaushanskaya et al. (2013) examined the interaction between phonological and semantic factors in novel word learning by probing the ability of adult native English speakers with different levels of Spanish exposure to learn novel word forms in an artificial phonological system. Participants learned phonologically familiar versus unfamiliar words mapped onto familiar referents (animals) or unfamiliar referents (aliens). The results showed that native English speakers with higher levels of Spanish experience outperformed less experienced Spanish learners in the wordlearning task where phonologically unfamiliar words were associated with familiar referents. The authors proposed that experience with a foreign language does not have to result in "full bilingualism" to facilitate novel word learning. Rather, it can still facilitate learning but only in situations that have been practiced during learning - that is, in learning tasks that resemble L2 acquisition-when phonologically unfamiliar words are learned in association with familiar referents. This suggestion echoes the framework we propose, wherein restricted experience with L2 learning improves language learning by virtue of direct implementation of prior learning strategies and experiences. At the same time, more elaborated experience with multilingualism may exert a global, indirect effect on language learning.

One way in which multilinguals may enjoy an indirect advantage involves enhanced cognitive control abilities, such as increased interference management abilities. Presumably, the prior experience of multilinguals has changed their cognitive linguistic system in a way that now facilitates novel language learning (see Indirect route, Fig. 1). Notably, not all cases where multilinguals outperform monolinguals in interference management reflect indirect effects. For instance, if the novel-learning task involves interference management of linguistic information, this could be viewed as direct transfer. To illustrate, Bartolotti and Marian (2012) trained English monolinguals and Spanish-English bilinguals on words in a novel artificial language, until a proficiency criterion was met.
Following learning, eye tracking and mouse tracking were used to measure cross-language interference during a spoken comprehension task. Eye and mouse movements to unrelated control items were contrasted with those to competing distractors, which overlapped phonologically between the L1 and the novel language. The results showed that monolinguals were more likely to visually fixate (and fixated for longer time) on cross-language competitors than on control items, but bilinguals were equally likely to attend to competitors and control items. The authors interpreted these results to suggest that bilinguals resolve competition from a known language earlier and more effectively than do monolinguals. They proposed that the bilingual experience improves the ability to manage cross-language interference by enhancing the ability to manage the activation of multiple languages and reducing interference from nontarget languages. Thus, extensive experience with managing multiple languages affects language learning through the enhanced ability to manage crosslinguistic interference. To the extent that prior experience and the novel learning situation both require interference management of linguistic representations, this should be viewed as direct effect, because multilinguals transfer previously practiced skills to the learning situation.

Therefore, for the multilingual advantage involving interference management to be considered an indirect effect, two premises should be accepted. One, that the experience of multilinguals with managing the simultaneous activation of their languages results in enhanced nonlinguistic interference management (e.g., Bialystok, Craik, Klein, \& Viswanathan, 2004). Second, that individual differences in nonlinguistic interference management predicts novel word learning. The first premise is currently debated in the field (e.g., Bialystok, Kroll, Green, MacWhinney, \& Craik, 2015; Paap, Johnson, \& Sawi, 2015; Valian, 2015), and the second awaits empirical investigation, but there are some suggestive evidence that individual differences in inhibitory control as measured by the Simon task does predict novel word-form learning under high interference conditions (Bartolotti et al., 2011, described below). Therefore, the possibility for indirect effects via enhanced interference management abilities awaits empirical support, but the results of Bartolotti and Marian (2012) do support a direct influence of prior experience with linguistic interference management.

Further support for the role of linguistic interference management abilities as directly promoting the multilingual advantage in novel language learning comes from a recent study by Bogulski et al. (in preparation). In their study, they examined whether the multilingual word-learning advantage depends on the language in which the words are being learned. They first replicated the advantage showing that EnglishSpanish bilinguals were more accurate than English monolinguals when learning Dutch words via English translations (L1 for both groups). Interestingly, they also examined Spanish- 
English and Chinese-English bilinguals and showed that when both bilingual groups learned the Dutch words via their L2 (English), no advantage was found. They thus concluded that the multilingual advantage in novel word learning is present only when the novel language is acquired via the native language (or dominant language) and suggested that this has to do with the pattern of language inhibition multilinguals have experienced. Specifically, during their past experience, Spanish-English and Chinese-English bilinguals had to inhibit their L1 in order to allow the acquisition of the L2 (English). Therefore, they may be less experienced in inhibiting their L2, as required in the word-learning task in order to map the novel form to the known concept. Further, the authors suggested that because the study was conducted in an English environment, in which multilinguals may experience the daily inhibition of their native language, it became especially difficult to inhibit English. These findings are in line with the suggestion that the multilingual advantage emerges when the requirements of the learning situation (in this case, inhibition of the L1) overlap with those previously experienced by the learner. Such an explanation focuses on direct implementation of practiced skills from prior learning experiences (experience with inhibition of a specific language) to the novel language learning scenario (for discussion, see also Bjork \& Kroll, 2015). Moreover, if this was an indirect influence through general inhibitory control abilities, multilinguals should have benefited from their prior experience with language inhibition in any learning situation including those that require inhibition of a different language from what they have practiced during learning.

Notably, a multilingual advantage did emerge in other studies in which bilingual participants learned through their L2. In Bartolotti and Marian's (2012) study, bilingual participants were presented with the novel words in the artificial language through their L2 (English). These seemingly contrasting findings can be settled in two (complementary) ways. First, the Spanish-English bilinguals in Bartolotti and Marian (2012) acquired their L2 at a relatively young age $(M=3.83)$, compared to the Spanish-English $(M=6.58)$ and Chinese-English ( $M=11.33$ ) in Bogulski et al. (in preparation). It is thus possible that the degree to which L1 inhibition was involved during L2 acquisition for Bartolotti and Marian's (2012) bilinguals was much reduced, or their pattern of dominance was less extreme, making it easier for them to inhibit the L2 in order to learn a novel language. Second, it is critical to keep in mind that the bilingual advantage in Bartolotti and Marian (2012) emerged during processing of the newly acquired information, and not during learning itself, because a learning criterion was imposed for all participants. It is still conceivable, then, that a multilingual advantage during learning is only possible when learning through the L1, making the overlap between prior experience and the learning situation high enough to allow direct implementation of previously practiced skills. This is not to rule out the possibility that part of the multilingual advantage in language learning is mediated indirectly through other cognitive and social factors which do not depend on such overlap.

Interestingly, the Bartolotti and Marian (2012) study makes the distinction between learning and access. In particular, the multilingual advantage in word learning could be attributed to either better learning of the novel words or to better access to the recently learned material during the test stage. Their results imply that (at least part of) the multilingual advantage is rooted in better access to the recently learned words because the training procedure ensured the same level of learning across groups. A similar pattern emerged in a recent study by Blumenfeld and Adams (2014). In that study, no difference was observed between Spanish-English bilinguals and English monolinguals on a nonverbal sound-to-symbol mapping task, which required mapping of tones to symbols based on pitch, timbre, and duration. This similar learning outcome for bilinguals and monolinguals was explained by the nature of the novel information. The authors suggested that relying on pitch and timbre cues was not central to bilinguals' previous experiences. It follows, then, that the bilingual advantage depends on substantial overlap between the characteristics of the novel information and the speakers' prior experiences. There were, however, subtle bilingual-monolingual differences in processing the newly learned material. When symbols were presented in combination with similar-sounding competitors requiring competition resolution mechanisms, bilinguals exhibited longer inhibition effects. Presumably, this difference is related to previous bilingual experience with cross-language competition resolution and supports the preposition above that multilingual experience with linguistic interference management directly enhances bilingual processing of the newly learned material. Thus, similar to Bartolotti and Marian (2012), these results indicate that even when learning outcome itself is equated, there can still be some differences in the nature of processing of the newly acquired information.

Thus, to more fully understand the mechanisms behind the multilingual effect on language learning, one must consider the potential effect of different factors not only on the acquisition phase itself and the learning trajectory associated with it, but also on the way different speakers process and utilize newly acquired information. Regardless of whether these effects manifest during learning or during processing, in both cases these could be viewed as direct effects of multilingualism on language learning because it is the similar experience with similar knowledge or with the given task that gives multilinguals their advantage. That is, multilinguals accumulate experience not only in word learning using similar tasks but also in processing multiple languages in parallel. Conversely, advantages during learning and during processing may result from indirect effects of multilingualism, via enhanced cognitive and social abilities. The studies thus far suggest such 
indirect effects are potentially mediated via the phonological system (Kaushanskaya \& Marian, 2009b), the lexicalsemantic network (Kaushakaya \& Rechtzigel, 2013), or via enhanced ambiguity processing (Kaushanskaya \& Marian, 2009a), but there is little empirical evidence for enhanced nonlinguistic inhibitory control mediating the novel word learning advantage. We return to this issue in the General Framework section.

Imposing a learning criterion (Bartolotti \& Marian, 2012) allows one to dissociate learning effects per se, from processing effects of the novel information. Laboratory controlled procedures, more generally, allow for reliable conclusions by verifying that multilinguals and monolinguals learn words under the same conditions. Further, different aspects of the learned material with relation to the known languages can be manipulated (for discussion of laboratory vocabulary studies, see Tokowicz \& Degani, 2015). In the real world, however, language learning is not as controlled as in the experimental context. Multilinguals and monolinguals often experience foreign language learning in the school system, and their differences emerge in such contexts as well. One study that examined these differences in the natural environment was conducted by Keshavarz and Astaneh, (2004), focusing on vocabulary production. They set out to examine the effects of multilingualism while varying literacy, by testing Armenian-Persian bilinguals who were literate in both of their languages and Turkish-Persian bilinguals who were only literate in Persian, and compared them to Persian monolinguals on productive abilities of English vocabulary. In this more natural study, the to-be-learned language (English) was learned outside of the experiment. They found that both bilingual groups outperformed the Persian monolingual group on the tested vocabulary learning. Moreover, the biliterate ArmenianPersian group numerically outperformed the monoiliterate Turkish-Persian group, suggesting literacy may provide additional support for vocabulary learning. We return to the role of biliteracy in the grammar (Sanz, 2000, 2007) and literacy acquisition (Schwartz, Geva, Share, \& Leikin, 2007; Schwartz, Kahn-Horwitz, \& Share, 2014) sections below.

To summarize, the adult literature on word learning suggests a robust multilingual advantage. This advantage could be the result of both direct and indirect factors, which can operate during learning and during processing of the novel vocabulary. Next we examine parallel effects among children.

\section{Children}

Only a handful of studies examined whether multilingual children differ from monolinguals in word learning. Moreover, novel word learning more generally has typically been investigated in children in the context of the mutual exclusivity assumption, which suggests that children prefer to maintain oneto-one mappings between labels and referents (Markman,
1990). Thus, when given a novel label, children will tend to associate it with an unknown referent rather than with a known referent for which they already have a label. This process is referred to as disambiguation, and it has been shown to develop in monolingual children around the age of 17 to 18 months (Byers-Heinlein \& Werker, 2009; Halberda, 2003; Markman, Masow, \& Hansen, 2003). Interestingly, multilingual children may differ in the developmental trajectory of this mutual exclusivity assumption due to their experience with multiple labels (one in each language) to one referent, and may use language membership cues to appropriately refrain from this assumption when they are sure the labels come from different languages (Au \& Glusman, 1990). Byers-Heinlein and Werker (2013) specifically examined the influence of infants' knowledge of translation equivalents (a label in each language to the same referent) on the development of disambiguation. In their task, English-Chinese bilingual infants (age 17 to 18 months) heard a novel label, and their looking toward a novel item versus a familiar item was taken as an index of disambiguation. The preference for the novel item was negatively correlated with infants' knowledge of translation equivalents. Specifically, bilingual infants who knew fewer translation equivalents tended to prefer novel items more than bilinguals who knew many translation equivalents, suggesting that the specific experience with one-to-many mappings directly influenced disambiguation when learning novel words.

Although as a group, bilingual infants at the age of 17 to 18 months did not exhibit the mutual exclusivity bias (ByersHeinlein \& Werker, 2013), they appear to exhibit this assumption later on, but their reliance on it is more flexible. Specifically, Kalashnikova et al. (2014) investigated differences between monolingual and bilingual children in their ability to accept lexical overlap while learning new words. Three-to-five-year-old children were presented with unfamiliar objects and introduced to either one novel label for one unfamiliar object (exclusivity condition) or to two novel labels for the same unfamiliar object (overlap condition). The results showed that whereas both bilingual and monolingual young children (under 4.5 years) relied on the mutual exclusivity assumption early on, with age differences emerged between the language background groups. In particular, the older monolingual children performed better than the older bilingual children in the exclusivity condition but performed worse than the bilinguals under the overlap condition. Thus, older bilingual children were better able to map two novel labels to a single unfamiliar referent. The fact that the difference emerged with age implies that the reduced reliance on the mutual exclusivity assumption develops once the speaker has accumulated enough experience in learning multiple labels (one in each language) to a single referent. Thus, multilinguals directly implement their flexible use of the mutual exclusivity assumption when required to learn many-to-one mappings of labels to referents later on. 
Although the focus in the mutual exclusivity studies is on the strategy children employ, it is interesting to consider these from the perspective of ambiguity learning. In particular, using a different paradigm, Kaushanskaya et al. (2014) asked children to map a novel label to either an unfamiliar referent for which no label exists (one to one; aliens condition) or to a familiar referent for which a label is already known (many to one; animals condition). They compared novel word learning of 5-to-7-year-old English monolingual children to that of classroom bilinguals with an average of 2 years of SpanishEnglish dual-immersion experience. The results showed a bilingual advantage only for familiar referents (many-to-one mapping; see also Kaushanskaya et al., 2013, with adults), leading the authors to conclude that classroom bilingualism has a specific impact on novel word learning, exerting an advantage only in learning situations that have been practiced before, namely mapping a novel label onto a known referent. Moreover, although Kaushanskaya et al. (2014) observed a bilingual advantage in verbal working memory as measured by the listening-span task, they reasoned that this was unlikely to enhance bilinguals' word-learning abilities (indirectly) because the bilingual word-learning advantage was constrained to learning with familiar referents only. Within the framework we present here, these findings stress the importance of direct influences that depend on the degree of overlap between prior experience and the learning situation. Thus, classroom bilinguals have gained experience in mapping novel labels to known referents, and this experience allows direct implementation in the new learning situation.

The interpretation of Kaushanskaya et al. (2014) for their data do not favor the role of indirect effects through working memory and executive control. Nonetheless, the contribution of these indirect sources to children's novel word learning has recently been highlighted. Yoshida et al. (2011) compared the performance of 3-year-old English monolingual children to a heterogeneous group of 3-year-old bilingual children in an artificial adjective learning task and a nonverbal attention control task. Whereas there was no difference between monolinguals and bilinguals in novel words associated with known adjectives, bilinguals outperformed monolinguals in learning novel adjectives (with no labels in any of the languages known) as well as on the attention control task. Learning novel adjectives was taken to depend on executive control because it requires inhibition of the typical tendency to interpret novel words as nouns (e.g., Markman, 1990). Positive correlations were indeed found between bilinguals' attentional control abilities and their performance on the novel adjective learning task, suggesting that children learning two languages employ enhanced executive control processes when learning novel words, perhaps more so than children growing up learning just one language. Further, the performance on the novel adjective-learning task was not linked to total vocabulary scores, indicating that it is not merely knowing more units of language but the experience with managing multiple languages that gives the multilingual children their advantage on the adjective-learning task. Within the framework we propose, these findings are in line with indirect effects of multilingualism on language learning because it is the enhanced cognitive ability of attentional control that puts multilingual children at an advantage over monolinguals. In addition, multilingual children did not know more adjectives than monolingual children in this study, implying that it is less likely that prior experience with learning adjectives directly influenced performance on this task.

This interpretation of Yoshida et al. (2011), that bilingual children, but not monolingual children, employ enhanced executive control processes when learning novel words, implicate differences between monolingual and multilingual children in the way they learn words. Brojde et al. (2012) compared bilingual 2.5-year-old children (speaking English and another language) to English monolingual children on their tendency to rely on pragmatic versus perceptual cues when mapping a novel label to a referent. Children were presented with a single novel label along with a single referent and were asked to extend this label to other referents based on perceptual similarity or on pragmatic cues. Brojde et al. (2012) found that when confronted with conflicting cues to word meanings, bilingual children tended to pay attention to pragmatic cues (i.e., the direction of the experimenter's eye gaze), whereas English monolingual children tended to pay attention to perceptual cues (i.e., the shapes of the objects). Conceivably, because bilingual children have experience with learning two labels for a given referent, they may need pragmatic cues to a greater degree relative to monolinguals in order to learn to map a label to a referent. Thus, multilingualism may influence the principles that constrain early processes of word learning. Notably, in this study there was no correct answer such that outcome learning measures could not be compared. Rather, the results are revealing with respect to the way novel words are learned and to the way multilingualism may affect this process. Multilingualism may change the weight given to different cues in the environment (pragmatic vs. perceptual), and these changes may lead to direct effects influencing learning in other contexts. To the extent that the balance between pragmatic and other cues turns out to influence performance of other linguistic features (beyond words), one can consider these pragmatic abilities as a mediating cognitive factor, but this issue has not been explored yet. In the context of word learning, this effect should be viewed as direct transfer of prior experiences onto the learning situation.

To summarize, the findings reviewed thus far demonstrate that multilingual children have an advantage in vocabulary learning in situations that require many-to-one mappings (Kalashnikova et al., 2014; Kaushanskaya et al., 2014). This advantage is evident for early multilinguals at a young age as well as for slightly older classroom multilingual children. 
Further, the multilingual experience may change the way in which novel words are acquired, shifting the weight toward pragmatic rather than perceptual cues (Brojde et al., 2012). Finally, speakers' attentional control may indirectly enhance their ability to learn novel words (Yoshida et al., 2011).

Overall, the literature suggests a robust advantage for multilinguals in vocabulary learning, especially for adults. This advantage appears to be rooted in both direct and indirect influences of multilingualism. In particular, direct effects are manifested when prior experience with similar learning tasks or materials makes future learning less novel. Such effects have been documented in both adults (Bogulski et al. (in preparation); Kaushanskaya \& Marian, 2009a; Kaushanskaya et al., 2013; Wang \& Saffran, 2014) and children (Kalashnikova et al., 2014; Kaushanskaya et al, 2014). Further, experience with cross-language competition that improves the ability to manage specific cross-language interference has been suggested for adults (Bartolotti \& Marian, 2012; Blumenfeld \& Adams, 2014; Bogulski et al. (in preparation). Finally, changes in the weight given to cues in the learning environment (such as enhanced reliance on pragmatic cue) appear to transfer to the novel word learning task in children (Brojde et al., 2012).

Interestingly, the multilingual advantage in vocabulary learning is unlikely to be reduced to such direct effects and is at least partially driven by indirect influences of multilingualism on the cognitive linguistic system. Specifically, multilingualism was suggested to indirectly influence adult word learning due to experience with more than one phonological system, arguably making the phonological system more flexible and better suited for absorbing novel phonological forms (Kaushanskaya \& Marian, 2009b; Wang \& Saffran, 2014). In addition, enhanced connectivity for overlapping representations and changes to the lexical-semantic network were suggested to explain the multilingual advantage in learning concrete novel words (Kaushanskaya \& Rechtzigel, 2012). Moreover, we speculated that multilingual experience with many-to-one mappings across phonological, orthographic, and semantic representations increases multilinguals' ability to manage ambiguity during learning (based on Kaushanskaya \& Marian, 2009a, 2009b; Kaushanskaya \& Rechtzigel, 2012). In children, enhanced attentional control abilities (Yoshida et al., 2011) were suggested as indirect mechanisms.

Next, we consider whether multilingualism entails any advantage in learning in other linguistic domains, namely, in phonology, grammar, and literacy.

\section{Phonology}

Phonology can be viewed as one aspect of novel word learning. In particular, word learning includes three componentslearning a new form (phonology), learning a new meaning, and learning the mapping between form and meaning (although in adult vocabulary learning the meaning itself is typically already known; see Sommers \& Barcroft, 2013). Although some of the previously reviewed literature addressed issues of phonology, the majority of these studies focused on learning the mapping between the novel phonological form and its meaning (be it familiar or unfamiliar, e.g., Kaushanskaya et al., 2013). Other studies, to be described next, focused on the effect of multilingualism on learning the phonological forms themselves.

Trembley and Sabourin (2012) examined learning of novel nonnative contrasts and compared the learning performance of English monolingual adults to English-French bilinguals and to multilinguals speaking English, French, and at least one other language. Results showed no difference among the groups in the ability to discriminate the novel nonnative contrasts before training. Critically, bilinguals and multilinguals showed improvement on contrast discrimination with training. Moreover, comparisons at posttest revealed a significant advantage for multilinguals over the monolingual group. Bilinguals exhibited a numeric nonsignificant advantage over monolinguals, but did show a significant advantage in measures of learning transfer. This multilingual advantage was attributed to what could be considered both direct and indirect sources. Specifically, the advantage may be the direct result of extensive experience with learning sounds in a foreign language, such that the learning situation is more familiar to multilinguals. This would be an example of a direct transfer of prior learning skills. At the same time, the advantage may stem indirectly from a general cognitive advantage such as enhanced phonological memory (see Kaushanskaya, 2012) and acoustic analysis abilities (Trembley \& Sabourin, 2012). These suggestions were not explored empirically.

Bartolotti et al. (2011) examined the possibility that the effect of multilingualism on novel phonological form learning is mediated by cognitive abilities of inhibitory control. Specifically, in a two-stage statistical learning procedure they manipulated the degree of interference experienced by the learners using an artificial novel language based on a Morse code. ${ }^{2}$ Successful learning of the Morse code required word segmentation based on statistical probabilities. They compared the performance of two groups of bilingual speakers with various language combinations divided by their language experience. Specifically, high-experienced bilinguals were characterized by higher L2 proficiency, earlier age of L2 acquisition, and higher frequency of L2 use in comparison to low-experienced bilinguals. The results showed that under low-interference conditions, high-experienced bilinguals were able to learn the Morse code whereas the low-experienced bilinguals were not. When interference was high due to competing cues to word boundaries, there was no difference

\footnotetext{
$\overline{2 \text { Because words }}$ were not assigned to meanings in this paradigm, we review this study under phonological learning rather than word learning.
} 
between the two bilingual groups. Instead, participants' inhibitory control ability as measured by the Simon task promoted successful word segmentation. The authors suggested a different contribution to each of the two factors: Whereas experience with language facilitated learning by increasing the ability to attend to statistical regularities in the signal, inhibitory control facilitated learning by increasing the ability to suppress conflicting language knowledge and to focus attention on the meaningful aspects of the novel words. Note that although inhibitory control facilitated word segmentation, this effect was independent of the effect of multilingualism. The results of this study imply that the effect of multilingualism on language learning works to enhance the ability to attend to statistical regularities. Within the framework we outline, this can be viewed as an indirect effect because experience with multiple languages boosted a cognitive ability, which served as a mediating factor for novel language learning. Note, however, that recent research suggests that statistical learning may not be a unified ability and thus successful implementation of practiced statistical learning abilities may vary with modality and input characteristics (Frost, Armstrong, Siegelman, \& Christiansen, 2015; Siegelman, Bogaerts, Chrisiansen, \& Frost, 2016; Siegelman \& Frost, 2015). Therefore, multilingualism may enhance auditory statistical learning abilities, for instance, but not necessarily visual statistical learning. The influence of multilingualism on statistical learning abilities, and its consequent indirect effect on language learning, remains a tentative suggestion at this point.

Antoniou et al. (2015) focused on learning phonetic contrasts in an artificial language varying the level of difficulty and the degree of similarity of the to-be-learned phonetic contrast to the participants' native languages. In the first experiment, English monolinguals were compared to MandarinEnglish bilinguals on their ability to learn Mandarin-like and English-like phonetic contrasts. Results showed that bilinguals outperformed monolinguals on both types of phonetic contrasts. Also, Mandarin-like contrasts were easier to learn by participants in both groups. In the second experiment, an additional group of bilinguals (Korean-English) was added, and Korean-like phonetic contrasts were learned along with Mandarin-like phonetic contrasts. Results showed that both bilingual groups outperformed the monolinguals on the Mandarin-like contrasts, but only the Korean-English bilinguals outperformed the other groups on the Korean-like contrasts. These findings show that the bilingual advantage is modulated by the difficulty of the phonetic contrasts of the novel language and by the similarity of these contrasts to the already known languages. Specifically, there was a phonetic learning advantage for bilinguals for contrasts that are universally easier (Mandarin-like), but language-specific experience may boost learning of universally difficult contrasts (Koreanlike), as demonstrated by the better performance of the Korean-English bilinguals.
Within the framework we propose here, these findings can be interpreted as supporting both direct and indirect effects. Indirect effects are reflected by the advantage observed for Korean-English bilinguals over monolinguals in learning Mandarin-like contrasts, although these bilinguals had no previous experience with such phonetic features. What particular indirect mechanisms support this advantage was not explored. In addition, direct effects were observed when only bilinguals with prior experience with Koreanlike contrasts outperformed the other groups on the Korean-like contrasts. Thus, these findings demonstrate two alternative routes to the multilingual advantage, which depend on the difficulty of the learning at hand. When learning is easy (e.g., easy to learn contrasts), the multilingual advantage is mediated indirectly through the cognitive linguistic system, but there is no evidence for direct effects due to language similarity. When learning is difficult, the indirect pathway is not sufficient to yield an advantage, and multilinguals may outperform monolinguals only through direct transfer of specific experiences.

Notably, because there was no monolingual Korean group in this study, it is difficult to determine whether the advantage for Korean-English bilinguals is solely based on experience with Korean, or whether their multilingual experience partially contributed indirectly to their advantage over English monolinguals. Moreover, the data in fact do not rule out the possibility that when difficult contrasts are learned, there is no multilingual advantage and that the advantage of the KoreanEnglish bilinguals is not unique to bilinguals.

In a related study, Wang and Saffran (2014) demonstrated a multilingual advantage in segmentation of words from a continuous input. Using an artificial tonal language that included syllable-level and tonal-level statistic regularities, the authors examined the contribution of previous experience with tonal languages and with multiple languages. Specifically, they compared the performance of adults from four groups: English monolinguals, Mandarin monolinguals, non-tonal language bilinguals and Mandarin-English bilinguals. The results demonstrated a multilingual advantage, such that the Mandarin-English bilinguals outperformed Mandarin monolinguals, and nontonal language bilinguals outperformed English monolinguals. Of interest, because the advantage for the nontonal language bilinguals cannot be explained by prior experience with lexical tone, the authors postulated an indirect effect of multilingualism on the statistical learning abilities of the speaker. Similarly, direct effects cannot explain the observed advantage for Mandarin-English bilinguals over Mandarin monolinguals because both groups had direct experience with similar contrasts. Thus, an indirect effect, perhaps via statistical learning abilities, is likely at play. This suggestion is in line with the proposal made above with reference to the Bartolotti et al. (2011) study, which demonstrated a multilingual advantage in learning a Morse-code-based language. 
At the same time, direct experience with specific linguistic cues (such as experience with tonal languages) does appear to make a contribution as well. This is because the Mandarin monolinguals were comparable in their performance to the nontonal language bilinguals. Thus, specific experience directly facilitate word-form learning, and a general experience with another linguistic system indirectly contribute as well.

Together, the reviewed studies suggest a multilingual advantage in novel phonological learning, even when phonology is to be learned with no associated meaning. Prior experience with specific phonological contrasts (Antoniou et al., 2015) or phonological cues (Wang \& Saffran, 2014) directly contribute to phonological learning. Moreover, prior familiarity with learning novel phonemes more generally (Antoniou et al., 2015; Trembley \& Sabourin, 2012; Wang \& Saffran, 2014) appear to contribute to the effect through indirect influences of multilingualism on the cognitive linguistic system and statistical learning mechanisms.

\section{Grammar}

To the extent that multilinguals gain direct experience in language learning and phonology, and that multilingualism influences cognitive and linguistic abilities that play a role indirectly in future learning, it is reasonable to expect effects of multilingualism beyond the lexicon, in grammar and literacy. Nonetheless, with the exception of a few classroom studies (Klein, 1995; Sanz, 2007), there is surprisingly little research in the domain of grammar.

Using self-report, Kemp (2007) compared adult multilinguals, experienced with two to 12 different languages, on their use of grammar learning strategies. The results show that speakers apply more grammar-learning strategies and use them more often the more languages they know. The author postulated that this is because experience with multiple languages allows one to automatize the use of grammatical strategies, thus freeing up resources to process other aspects of the input, speeding up the acquisition of additional languages. These findings reveal differences in the way multilinguals approach grammar learning but do not provide evidence with respect to the learning outcome. Nonetheless, to the extent that multiplicity of learning strategies result in better learning, this exemplifies an indirect effect of multilingualism by which experience with multiple languages expands the learning tools available to the learner by freeing up cognitive resources.

Some support for a difference in grammatical learning outcome between monolinguals and multilinguals comes from an early study, in which Klein (1995) observed that adolescent multilinguals outperformed monolinguals in learning English as a foreign language, in both the lexical and the syntactic domain. Specifically, in a grammaticality judgment and correction task highlighting verbs and their complimentary preposition (e.g., wait for, play with), multilinguals were better able to judge whether a given sentence was acceptable in English (e.g., The girl wait the bus) and to properly correct it, in comparison to monolinguals. Heightened metalinguistic skills and enhanced lexical knowledge as well as a less conservative learning procedure were suggested to stand at the core of these advantages. Thus, the author speculated (but did not corroborate) that multilingualism indirectly affects grammar learning via linguistic and metalinguistic skills as well as multiplicity of learning strategies.

Additional evidence for the effects of multilingualism on grammar learning comes from a study by Sanz (2007), in which she examined adolescent Catalan-Spanish bilinguals on an English proficiency test (see also Sanz, 2000). The results demonstrated a positive relationship between balanced abilities to read and write (i.e., balanced biliteracy) in Catalan and Spanish and the performance on the grammar section of the English test. On the vocabulary section, however, no relationship was observed between balanced literacy abilities and performance. Thus, only grammatical abilities were found to correlate with biliterate experience. Moreover, balanced oral proficiencies did not correlate with the grammar or vocabulary performance. Notably, because there was no comparison to a monolingual control group, it is unclear whether multilingualism per se exerts an influence on grammar learning. Further, although suggestive of a difference in grammar learning due to biliterate experience, these findings provide little insight into the mechanism that might be at play. Whether these advantages come from direct transfer of experience with similar grammatical constructions, or from indirect enhanced cognitive and linguistic abilities remains unknown. Similarly, Abu-Rabia and Sanitsky (2010) found that Russian-Hebrew biliterate sixth-graders outperformed Hebrew monolinguals on an English syntactic judgment task, but the independent contribution of multilingualism (without multiliteracy) was not examined.

In a study with Spanish-English biliterate adults, Stafford, Sanz, and Bowden (2010) examined the effect of L2 (English) age of acquisition (AoA) on learning the grammar of an L3 (Latin). The results revealed no difference between early $(\mathrm{AoA}=8.3)$ and late bilinguals $(\mathrm{AoA}=25.1)$. As in the Sanz (2007) study described above, because these groups were not compared to monolingual controls, it is difficult to determine whether multilingualism had any impact on novel grammatical learning.

Thus, there is very little research on differences between monolinguals and multilinguals in learning the grammar of a novel language, and, although suggestive of a multilingual advantage, systematic research in this domain is much needed. Further, how this advantage, if found, develops across the life span is currently unknown. 


\section{Literacy}

The link between multilingualism and literacy learning has received some attention, mostly in the field of initial literacy acquisition in children. In most of these studies, however, it was multiliteracy rather than multilingualism that was examined in relation to novel literacy acquisition. We focus here on studies which explicitly compared multilingual to monolingual speakers, though the literature on the role of biliteracy in L3 literacy acquisition is more extensive (e.g., Errasti, 2003).

Focusing on measures of reading comprehension, Van Gelderen et al. (2003) did not observe a multilingual advantage in English reading when comparing adolescent Dutch monolinguals and Dutch bilinguals speaking another language. The monolingual group in the study performed significantly better than the bilingual group on reading proficiency and sentence verification tests in English. No group difference was observed on tests of word recognition and vocabulary, grammatical, or metacognitive knowledge. The authors suggested that because the bilingual participants were monoliterate (in Dutch only), they did not differ from their monolingual counterparts. Thus, there may be no literacy advantage associated with spoken multilingualism. Notably, the bilingual group in this study also scored lower on a reading proficiency test in Dutch, suggesting that they may be weaker readers in general and that this may be the reason they did not outperform the monolinguals on the English test as well (but see, e.g., Gollan, Montoya, Cera, \& Sandoval, 2008; Ivanova \& Costa, 2008, showing reduced linguistic abilities for bilinguals compared to monolinguals even in their dominant language). Further, potential socioeconomic status (SES) confounds were not controlled for.

Support for the dissociation between multilingualism and multiliteracy in their effect on literacy acquisition comes from a series of studies by Schwartz and colleagues. Specifically, Schwartz et al. (2007) examined the impact of literacy in the L1 on the acquisition of English literacy skills, which served as an L2 for monolingual participants and as an L3 for bilingual participants. Three groups of 11-year-old children were compared on literacy, linguistic, and metalinguistic measures in three languages: Russian, Hebrew, and English. Results showed that biliterate Russian-Hebrew bilinguals outperformed Hebrew monolinguals and monoliterate Russian-Hebrew bilinguals (who are literate only in their L2, Hebrew), on three basic literacy skills in English: phoneme analysis, nonword decoding, and spelling. In a related study, Kahn-Horwitz, Schwartz, and Share (2011) observed that Russian-Hebrew biliterates similarly outperformed Hebrew monolinguals and monoliterate Russian-Hebrew bilinguals on several English decoding and spelling tasks (of short vowels and consonant clusters). In both studies, the authors took their findings to suggest that cross-linguistic transfer of literacy skills can be found even across different alphabetic orthographies. They further suggest that literacy in the native language improves L3 literacy acquisition but that this is in part because of the similar orthographic principles of Russian and the target language, English. Critically, in a regression analysis in Schwartz et al. (2007), bilingualism as a separate factor from biliteracy, did not contribute significantly to reading accuracy in English, and in fact, the monoliterate bilingual group often scored lower than the monolinguals. This lack of multilingual effect on literacy acquisition stands in contrast to the above reviewed findings on the multilingual effect on word learning. Novel word learning (i.e. English vocabulary acquisition) was not directly measured in this study.

A study with participants from a similar population of Russian immigrants to Israel, assessed self-teaching of orthographic conventions in English as an L3 (Schwartz et al., 2014). Three groups of sixth-graders (10-year-old children), who have had English instruction in school for 4 years prior to the study, were compared on a self-teaching task. During a learning phase, participants (Russian-Hebrew biliterates, Russian-Hebrew monoliterates, and Hebrew monolinguals) were exposed to homophonic English words embedded in short texts. At test, biliterate bilinguals outperformed both monoliterate bilinguals and monolinguals in English-naming accuracy, naming speed, and orthographic choice on the homophonic English words previously encountered in the text. Schwartz et al. (2014) concluded that the experience with another orthography facilitated Russian-Hebrew biliterates' ability to learn orthographic conventions in English. Again, this interpretation was suggested to result from the orthographic proximity between Russian and English as opposed to Hebrew and English.

Notably, mutliliteracy may provide an advantage not only through direct transfer due to orthographic similarity but also through alternative indirect pathways (for such an interpretation, see Abu-Rabia \& Sanitsky, 2010). It is possible that experience with two orthographic systems may allow an efficient learning of new orthographic conventions due to a general more flexible orthographic system. This suggestion is similar to the one made by Kaushanskaya and Marian (2009b) regarding the allegedly more flexible phonological system. In particular, a more flexible orthographic system would be one in which the mapping of orthography to phonology is less strict allowing, for instance, a single letter to be mapped onto two sounds (e.g., the letter $H$ corresponding to the sound/n/in Russian). More generally, experience with multiliteracy may advance the speakers' ability to accept additional alternative writing systems to represent spoken language.

The contribution of direct and indirect sources for the multiliteracy advantage in novel literacy acquisition remains to be tested empirically. For instance, evidence for indirect 
effects could come from studies that neutralize the direct pathway by making the to-be-learned language dramatically different from the languages known by the participants (e.g., Mandarin-English bilinguals learning Hebrew). In such a scenario, any observed difference could only result from indirect effects because direct transfer of orthographic principles is not available.

A related approach was adopted by Kahn-Horwitz et al. (2014), who examined English learning by 10-year-old children, comparing Hebrew monolinguals to CircassianArabic--Hebrew multilinguals. The two groups differ not only on the number of oral languages spoken but also in literacy knowledge, such that the multilingual group is biliterate in Arabic and Hebrew. Interestingly, they examined acquisition of orthographic conventions that are shared or different across the relevant languages. They found that when the to-belearned (English) convention is present in one of the languages known to the bilterates, an advantage emerged over monolinguals. However, for conventions that are truly novel for all participants, no biliteracy advantage emerged. Thus, the biliterate children outperformed the monolingual children in decoding and spelling of almost all English conventions, but this advantage did not extend to the case of silent $<\mathrm{e}>$, which does not exist in any of the orthographies known to the biliterates. Notably, this specific convention is a difficult convention to learn even for native English speakers (Davis \& Bryant, 2006). Nonetheless, these findings provide strong support to the suggestion that multiliteracy contributes to literacy acquisition via direct transfer of available (more elaborate) knowledge.

In a study assessing writing, reading, and speaking proficiency in French as a foreign language in the Canadian context, Mady (2014) compared monolingual children in the sixth grade to their bilingual peers. The study included two bilingual groups: immigrants who arrived in Canada during elementary school and lifelong bilinguals, both of whom spoke English as their L2 with various L1s. Both bilingual groups scored higher than the monolinguals on the writing section of a French proficiency test. However, on the speaking and reading sections, an advantage was found only for the immigrant bilingual group, with no difference between the lifelong bilinguals and the monolinguals. Based on self-report, and using a regression analysis, the authors showed that these bilingual advantages were not due to metalinguistic awareness, strategy use, or proficiency in L1 or English. Instead, social predictors were found to be more influential, including willingness to communicate and anxiety towards French. Thus, a bilingual advantage emerged in novel language writing, but the advantage in speaking and reading was restricted to immigrant (rather than lifelong) bilingual children. The highlighted social predictors could be viewed as reflecting indirect effects of multilingualism on language learning.
The findings reviewed above show that multilingual children learning novel language literacy enjoy an advantage over monolinguals only to the extent that multilingualism entails additional characteristics. Several studies highlight the role of multiliteracy (Abu-Rabia \& Sanitsky, 2010; Kahn-Horwitz et al., 2014; Kahn-Horwitz et al., 2011; Schwartz et al., 2007; Schwartz et al., 2014; Van Gelderen et al., 2003), whereas the study by Mady (2014) suggests social factors associated with immigration underlie these effects. Furthermore, the available evidence suggest that multiliteracy affects novel literacy learning through direct transfer of prior knowledge (Kahn-Horwitz et al., 2014; Schwartz et al., 2007; Schwartz et al., 2014). Notably, all of these studies focused on children's literacy acquisition.

In adults, one study found an advantage in reading comprehension for Turkish-Persian bilinguals compared to Persian monolinguals (Modirkhamene, 2006). The groups were tested on English reading comprehension tests several times over 2 years of their academic studies. Results showed that bilinguals scored higher than monolinguals across time. The mechanisms beyond this advantage were not explored empirically. More generally, the influence of multilingualism on adult literacy learning awaits future investigation.

\section{Direct and indirect effects—General framework}

The studies reviewed above examined the contribution of multilingualism to language learning across several language domains, including vocabulary, phonology, grammar, and literacy. The framework we propose views this multilingual contribution as stemming from both direct and indirect sources. Direct effects are those that transfer "as is" from prior experience to the learning situation, and critically depend on the similarity between early experiences and novel language learning in task demands and the to-be-learned materials. These include overlapping pieces of knowledge as well as previously practiced strategies and skills. Indirect effects are those that involve the cognitive and social abilities of the learner and involve mediating abilities that have changed due to prior experiences and are now influencing novel learning. The weight given to direct and indirect pathways may change as a function of learner and language characteristics. In what follows we highlight these potential modulations.

\section{Speakers of different ages (adults vs. children)}

The age factor has been a prominent dimension in the literature reviewed above, such that individual studies either examined novel language learning among children or adults, but not both. Nevertheless, comparisons across this literature suggest that throughout the life span, both direct and indirect effects of multilingualism are documented, especially in the 
domain of word learning. In particular, vocabulary-learning studies show that direct effects are rather stable across the life span, such that the specific experiences that are being transferred are the same for children and adults. As early as 17 to 18 months, children begin to exhibit the influence of prior experience with mapping two labels to a single referent, and this prior experience with many-to-one mappings facilitate learning in future ambiguous situations for both children and adults. Additional direct mechanisms were postulated for different age groups. In particular, for adults, direct experience with managing linguistic interference was hypothesized to transfer to novel word learning, whereas for children reliance on pragmatics over perceptual cues was highlighted. The indirect effects hypothesized for different ages are not overlapping. Specifically, for adults the advantage was suggested to stem from flexibility of the phonological system and the organization of the lexical-semantic system. Further, increased statistical learning abilities were highlighted as a potential mediating factor. Moreover, we speculated that heightened abilities to manage ambiguities between semantic, phonological, and orthographic representation may partially contribute to the multilingual advantage in word learning in adults. In children, in contrast, research provides evidence for increased attentional control abilities (based on Attention Network Test performance; see Yoshida et al., 2011) as a potential indirect mediator of the multilingual word-learning advantage, but in adults such executive function mediators were often postulated but were rarely empirically examined. Bartolotti et al. (2011) did not observe a multilingual advantage in executive function as measured by the Simon task but did document a relation between performance on the Simon task and learning of novel word forms.

In phonology learning, the literature is focused exclusively on adults, providing little opportunity to examine cross-age effects. For these adult populations, both direct transfer of prior experience with phonological contrasts and cues and more indirect effects through enhanced statistical learning abilities were postulated (Bartolotti et al., 2011; Wang \& Saffran, 2014). In grammar and literacy learning, the literature is heavily skewed toward children, with very little research on adults. Moreover, the particular mechanisms beyond the group differences were seldom explored. Multiliteracy was emphasized as an underlying factor, but this dimension could operate directly through orthographic similarity and/or indirectly through more general cognitive and social abilities. The results of the study by Kahn-Horwitz et al. (2014) shed some light on the interplay between direct and indirect effects. Specifically, Kahn-Horwitz et al. (2014) showed, within participants, that the multilingual advantage was limited to items that included ortho-phonological conventions that were present in the other languages of biliterate children. Thus, greater weight was ascribed to direct sources. Nonetheless, the contribution of indirect sources, such as enhanced statistical learning (highlighted in the vocabulary-learning literature) or metalinguistic awareness (highlighted as a consequence of biliteracy; e.g., Bialystok, Luk, \& Kwan, 2005, and of multilingualism: Herdina \& Jessner, 2002), were not explored.

\section{Multilingual language background}

An important dimension to consider when examining the effect of multilingualism on language learning is the language background of the learner. In particular, differences in L2 age of acquisition, in L2 learning circumstances and in the ultimate L2 proficiency of the learner, may all contribute to the pattern of novel language learning. Critically, these components are tightly linked, and it is often difficult to isolate effects that are due to early versus late age of acquisition, classroom versus environment-based learning, and or high versus low proficiency. Of the studies reviewed above, the circumstances of prior language learning were the factor that received most attention, as detailed below.

\section{L2 learning circumstances (environment vs. classroom)}

The circumstances of prior language learning are especially important to consider under the framework we propose. Specifically, to the extent that direct effects depend on the similarity between prior language learning and the novellearning situation, multilinguals who acquired their languages through formal classroom-based instruction are expected to differ from environment-based multilinguals (both lifelong and immersion-based) who acquired their languages in a more natural context. In drawing these distinctions, however, it is important to keep in mind that not all classroom-based and not all environment-based multilinguals are the same. Dualimmersion classroom programs, for instance, may differ from typical language-classroom instruction environments, and similarly lifelong multilinguals may differ from immigrant populations in important social motivational factors (Mady, 2014).

The difference between classroom-based and environmentbased multilinguals was explicitly addressed in the vocabulary learning literature, where Kaushanskaya and Marian (2009a, 2009b) demonstrated that the multilingual advantage documented for adult classroom-based bilinguals (Papagano \& Vallar, 1995; Van Hell \& Candia Mahn, 1997; see also Kaushanskaya et al., 2013) was present for adult environment-based bilinguals as well $(\mathrm{AoA}=5.44$ and 2.21). Similarly, in children, the direct effect of experience with one-to-many mappings contributed to the multilingual advantage in word learning for classroom-based bilinguals (Kaushanskaya et al., 2014, AoA = 4.34) as well as for lifelong bilingual children (Kalashnikova et al., 2014). Note, however, that these parallels are drawn based on informal 
comparisons across studies, because no study to date directly compared the two populations.

The controlled studies described above examined novel language learning in situations that resemble classroombased multilingualism. As a result, classroom-based multilinguals could utilize their prior experience with the learning situation and rely more on direct implementation of these practiced skills. In the few studies that examined this classroom-based population, there was no evidence of indirect effects (Kaushanskaya et al., 2014). At the same time, in such controlled studies, environment-based multilinguals likely outperformed monolinguals mostly through indirect effects mediated through cognitive abilities (Yoshida et al., 2011), because the novel learning situation did not resemble their prior experience. However, if and when novel language learning takes place more naturally (e.g., in an unstructured environment-based context), we may expect environmentbased multilinguals to utilize their prior experiences more directly and to thus enjoy both direct and indirect benefits. In such natural language learning contexts, it is unclear whether classroom-based multilinguals will enjoy any advantage over monolingual speakers, given that it would require indirect mediation, which to date has not been documented for classroom-based multilinguals. These conjectures assume that indirect effects are always available to influence performance but that direct effects inherently rely on the similarity across prior and present learning circumstances and materials.

In phonology learning, both classroom-based multilinguals (Trembley \& Sabourin, 2012) and environment-based multilinguals (Antoniou et al., 2015; Wang \& Saffran, 2014) were compared to adult monolinguals. For both types of populations, there appears to be a multilingual advantage in novel phonology learning. Nonetheless, direct comparisons across L2 learning circumstances await future studies.

The few studies that examined novel grammar learning do not provide much evidence with respect to the contrast between classroom-based and environment-based multilinguals, partly because very few details are provided regarding participants' language background. In literacy learning, the distinction between classroom-based and environment-based multilinguals becomes more complex. In particular, multiliterate Russian-Hebrew speakers (tested in Abu-Rabia \& Sanitsky, 2010; Kahn-Horwitz et al., 2011; Schwartz et al., 2007; Schwartz et al., 2014) learned both languages in an immersion-based context (through immigration to a Hebrew speaking environment), but it appears that it is their prior literacy that played the prominent role in their novel literacy learning advantage. Notably, orthography learning takes place in structured contexts, and thus these environment-based multilinguals enjoyed an advantage through their classroombased ability. Moreover, other research suggest that environment-based multilingualism by itself (without classroom-based experiences) does not seem to enhance literacy acquisition in a new language (Schwartz et al., 2007). However, it is unclear whether the classroom-based experiences alone, without additional substantial environment-based learning, would result in advantages in novel literacy learning. For instance, would classroom-based French-English bilinguals outperform French monolinguals in literacy learning of a novel language (e.g., Russian). Future research would therefore have to explore whether the multiliteracy effects could be observed for classroom-based multilinguals (mono- or multiliterates).

\section{L2 age of acquisition (early vs. late AoA)}

Of the reviewed studies, one study compared the performance of early versus late bilinguals in novel language-learning (Bartolotti et al., 2011). They showed that early bilinguals $(\mathrm{AoA}=5.4)$ outperformed late bilinguals $(\mathrm{AoA}=12.3)$ in word-form learning under low interference conditions. When interference was high, inhibitory control but not L2 AoA influenced behavior. Importantly, in the sample tested L2 AoA was correlated with proficiency, such that early bilinguals were also more proficient in their L2 than late bilinguals. As such, it is not possible to disentangle the contribution of these two language background dimensions. In the grammatical domain, one study by Stafford et al. (2010), which examined the effect of L2 AoA, observed no difference between early (AoA $=8.3)$ and late bilinguals $(\mathrm{AoA}=25.1)$. Notably, because these groups were not compared to monolingual controls, it is difficult to assess based on this study whether multilingualism had any impact on novel grammatical learning. Moreover, even when performance is equal across early and late multilinguals, there may be underlying differences as a function of AoA in the mechanisms at play. To illustrate, as alluded to earlier, multilinguals who acquired their L2 early in life may have gained substantial experience in inhibiting not only their L1 but also their L2. Thus, when faced with novel language learning they may be able to learn the novel language through their L2 and not only through their L1, because they can utilize inhibition on both languages (Bogulski et al. (in preparation).

\section{Current proficiency and use}

As mentioned above, multilinguals' proficiency (and or AoA) influenced word-form learning under low interference conditions (Bartolotti et al., 2011). Further, Kaushanskaya et al. (2013) focused on the contribution of multilinguals' L2 proficiency and use to performance in novel language vocabulary learning. They found that learners who performed better on the task (learning to map a novel phonological form onto a familiar referent) were those with higher current L2 proficiency and exposure. No monolingual comparison was included, 
and thus it remains unclear whether multilinguals with lower current L2 proficiency and use still outperform monolinguals.

Because few studies examined the contribution of language background characteristics, and the independent contribution of each dimension (learning circumstances, AoA, proficiency) was not contrasted, it is difficult to identify their unique role. Further, these dimensions are likely to influence not only ultimate learning performance but also the way in which novel language learning is approached. Future studies that directly contrast the contribution of these dimensions by including diverse participant populations could shed light on these mechanisms.

\section{Number of known languages (bilinguals vs. trilinguals)}

As reflected in this review, we consider multilingualism to be a multidimensional space, with various influencing factors. One such factor is the number of languages known by the speaker, such that monolinguals may differ from bilinguals who in turn may differ from trilinguals, and so on. Nonetheless, the majority of the studies reviewed here drew a distinction between monolinguals (one language) and speakers of more than one language. Thus, most studies compared monolinguals to bilinguals, but a few compared monolinguals to multilinguals (Klein, 1995; Kahn-Horwitz et al., 2014; Van Hell \& Candia-Mahn, 1997). Moreover, a handful of studies directly compared bilinguals to multilinguals (Papagano \& Vallar, 1995; Trembley \& Sabourin, 2012; Kemp, 2007; see also Byers-Heinlein \& Werker, 2009) and observed an advantage for multilinguals over bilinguals (at least in some measures; see Trembley \& Sabourin, 2012). This pattern of results underscores the continuous nature of this dimension and calls for future studies to address this issue more explicitly (see, e.g., Herdina \& Jessner, 2002).

\section{Language similarity}

The degree of overlap between the languages of the speakers may play a prominent role in the pattern of transfer when learning a novel language (for discussion, see also Linck, Michael, Golonka, Twist, \& Schwieter, 2015). Two aspects of this dimension should be considered. First, the similarity of the novel language to the languages known by the speaker will influence the degree of direct transfer. This is because if the novel language resembles the available knowledge of the speaker (e.g., in phonological contrasts, in grammatical structures), there are more opportunities for direct transfer. Indeed, Schwartz et al. (2007; Schwartz et al., 2014) suggested that because the to-be-learned language (English) resembled the orthography of one of the known languages of multilingual speakers (Russian of Russian-Hebrew bilinguals), multiliterate children outperformed mono-literates in learning literacy in the novel language. Interestingly, the effect of language similarity may surface when the to-be-learned material is more difficult to learn. Antoniou et al. (2015) showed that Korean-English bilinguals outperformed MandarinEnglish bilinguals when learning more difficult phonological contrasts (those that resembled Korean), but MandarinEnglish bilinguals did not outperform the Korean-English bilinguals when learning the less difficult to learn (Mandarin-like contrasts).

Second, the similarity of the known languages of the multilingual speakers to each other may influence the organization of the linguistic and cognitive system. Specifically, this may operate in two complementary ways. One, multilinguals of less similar languages (e.g., Kahn-Horwitz et al., 2014) enjoy a greater probability that the to-be-learned language will resemble at least one of the languages they know, enhancing the opportunity for direct transfer. Two, the cognitive system of multilingual speakers may be differentially influenced by multilingualism as a function of language similarity, and this may affect the opportunity for indirect influences on novel language learning. In particular, when the different languages of multilinguals overlap greatly, it may be less advantageous for the speaker to inhibit one language when using the other. A similar suggestion was raised by Van Assche, Duyck, and Gollan (2013), who demonstrated in a phoneme fluency task local inhibition (i.e., item specific) for both Chinese-English and Dutch-English bilinguals, but global language inhibition for Chinese-English bilinguals only. They postulated that because Dutch and English have many cognate words (words that overlap in both form and meaning across languages), it becomes less beneficial to inhibit the nontarget language. Following the same logic, one can propose that multilinguals of languages which differ dramatically in grammatical structures, for instance, would benefit from decreasing the activation of the nontarget language more than speakers of grammatically similar languages. If this is the case, then the degree of language similarity would affect the organization of the cognitive-linguistic system and could thus affect the potential for indirect influences of multilingualism on novel language learning.

These considerations predict that multilinguals of languages that are less similar should enjoy a greater advantage in novel language learning than multilinguals of similar languages. Such an advantage could operate through both direct and indirect pathways. However, it is also possible that multilingual speakers of similar languages are routinely required to manage cross-language interference more than multilingual speakers of less similar languages (for a similar argument, see Linck, Hoshino, \& Kroll, 2008). As a result, similar language multilinguals may be better equipped with cross-language interference management capacity, which may directly promote novel language learning.

Few studies compared different groups of multilinguals within the same study. In the domain of vocabulary learning, 
Bogulski et al. (in preparation) and Kaushanskaya and Marian (2009b) compared Mandarin-English to Spanish-English bilinguals, and Wang and Saffran (2014) compared Mandarin-English to nontonal language-English bilinguals. These particular studies do not provide strong support for the above predictions. Specifically, bilinguals of languages that are more similar (Spanish-English or nontonal languagesEnglish) did not differ from bilinguals of less similar languages (Mandarin-English) in vocabulary learning (Kaushanskaya \& Marian, 2009b) and word segmentation (Wang \& Saffran, 2014). In one case Bogulski et al. (in preparation), in addition to language similarity, the two bilingual groups also differed in AoA, L2 proficiency, and performance on cognitive measures, making direct comparisons less informative. In two additional studies, comparisons were made across bilingual groups, but because additional factors were manipulated at the same time (the similarity of the to-belearned language was manipulated, Antoniou et al., 2015; the literacy background of the participants, Keshavarz \& Astaneh, 2004), one cannot isolate the effect of language similarity in these studies. This issue, therefore, awaits future work.

\section{Interactions within and among dimensions}

So far, we have discussed the different pathways to the multilingual advantage in novel language learning as orthogonal dimensions. In fact, it is relevant to consider the interaction between the direct-indirect dimension and that of learning versus knowledge of multiple languages. Specifically, direct effects are those that depend on the similarity between past experiences and current requirements. Notably, these past experiences could have accumulated during the learning process itself, or during dual-language processing. For instance, classroom bilinguals have gained experience in explicitly learning vocabulary, such that when they are faced with a similar task they can easily implement what they practiced (e.g., EnglishSpanish classroom bilinguals required to map a novel phonological form onto an existing orthographic representation; Kaushanskaya \& Marian, 2009a). Critically, past experiences could also be those practiced during (dual) language use. Therefore, as noted by Bogulski et al. (in preparation) proficient multilinguals have practiced inhibiting their dominant language when using their less dominant language. Therefore, only when they learn a novel language via their dominant language, multilinguals could capitalize on their past experience and exhibit advantages over monolinguals (but see Bartolotti \& Marian, 2012).

To dissociate whether the critical experience was gained during learning or during dual-language use, one can capitalize on cases of switched-dominance multilinguals. For instance, a native Spanish speaker who learned English as a second language experienced the need to inhibit the L1
Spanish during learning. However, if this bilingual is now more dominant in English, she may be experienced in inhibiting the dominant language, English, in order to communicate in Spanish. When learning a novel language, she may benefit from prior experience during a similar learning situation and thus be better at learning via the L1 Spanish. Alternatively, she may benefit from prior experience in inhibiting the dominant language during dual-language use and thus may be better at learning via the dominant language English. Future studies could shed light on this issue.

Similarly, indirect effects could evolve from past experiences in learning and/or processing of multiple languages. For instance, prior experience in learning a second language could have promoted multilinguals' statistical learning abilities (Wang \& Saffran, 2014), such that when faced with novel language learning, multilinguals are better at attending to statistical regularities in the input, even when using different modalities or cues. Further, prior experience in processing and negotiating the use of multiple languages could have promoted multilinguals' attentional control, such that multilinguals are better than monolinguals at inhibiting distracting information when learning a novel language (Yoshida et al., 2011).

As mentioned earlier, multilingualism may affect not only the ultimate level of performance (i.e., learning outcome) but also the way in which such knowledge is acquired (e.g., reliance on pragmatic vs. perceptual cues; Brojde et al., 2012). Further, once this knowledge has been acquired, the speaker is required to develop fluent use of this knowledge in communicative settings. The studies reviewed above mostly focused on the first component (acquiring the knowledge) with little research done on processing of the newly acquired information (but see Bartolotti \& Marian, 2012; Blumenfeld $\&$ Adams, 2014). Future investigations should examine whether multilingualism affects how the newly acquired material is integrated and used in more contextualized production and comprehension settings.

Finally, in the framework we propose, direct and indirect effects are viewed as alternative pathways by which multilingualism may affect novel language learning. The distinction between these two pathways is determined based on the degree of similarity between prior experiences and the novel learning situation. Critically, however, we do not view this distinction as a true boundary between the two pathways. Rather, it may be more informative to think of direct and indirect effects as falling along a continuum, ranging by the degree of generalization needed. Thus, transfer is more direct the greater the resemblance between the novel learning situation and prior experiences, and becomes less direct (indirect) as the novel learning situation is more novel. When the novel situation imposes new constraints, the learner is required to capitalize on more (domain) general cognitive and linguistic abilities. For instance, if experience in one language domain 
(e.g., literacy) affects learning of another language domain (e.g., vocabulary learning) general mechanisms must be at play (Keshavarz \& Astaneh, 2004). To appreciate the role of generalization, consider for example the contribution of prior experience with mapping two labels onto a shared referent. As suggested earlier, such prior experience may work directly to facilitate novel vocabulary learning in a situation including competition (see overlap condition; Kalashnikova et al., 2014). However, to the extent that experience with ambiguous mappings influence the organization and flexibility of the linguistic system more generally, multilinguals may be able to accommodate different types of ambiguities in future learning. Thus, they may be better at learning synonymous words (two labels to a shared meaning) and homonyms (one label with two meanings). Such influences are still constrained within the lexical-semantic (linguistic) system and could thus be viewed as less indirect than executive function but are clearly not mere copying of prior experiences into novel language learning.

\section{Future directions}

The current review points to several areas in which more research is needed. For one, the effect of multilingualism on grammar learning among adults is currently unknown. Moreover, the studies suggesting a multilingual advantage in this domain for children provide very little insight into the mechanisms beyond such effects. Thus, there is a clear need for studies systematically examining if and how multilingualism influences novel language grammar learning.

In addition, the literature is focused on isolated outcome measures, where learners are tested for their knowledge of the newly acquired information in simple recognition and recall tests. Future studies should expand on this and test how learners integrate and use the newly acquired knowledge to communicate effectively. Thus, more complex tests that require learners to use words in sentences, in both production and comprehension tasks, would advance our understanding greatly. Such complex tasks are more ecologically valid, as language processing naturally involves more than one component at a time. Nonetheless, the use of more complex tasks makes it difficult to isolate the contribution of specific language components. Thus, an approach that adopts more complex tasks would be most informative in cases where our understanding of specific components is more established (i.e., word learning) rather than in subdomains where we know very little about the multilingual effect (e.g., grammar).

Moreover, multilingualism affects not only the learning of novel language vocabulary but also processing of this newly acquired information (e.g., Bartolotti \& Marian, 2012). Prior processing studies focused on comprehension tasks, but more work is needed in the production modality. Namely, it is currently unknown whether multilinguals outperform monolinguals when producing the newly acquired information in communicative settings. It could be the case that multilinguals would suffer greater interference from all available representations (i.e., from all languages they know) in comparison to monolinguals. Conversely, it could be that multilinguals' experience with managing competition would allow them to more efficiently negotiate the simultaneous activation of their languages in comparison to monolinguals that only now became bilinguals. Thus, future research would shed light on whether during production, experience in managing competition outweigh the increased competition for multilinguals. These issues resemble comparisons of multilinguals versus bilinguals in language processing more generally.

We suggest that the direct and indirect effects of multilingualism on language learning are modified by learner and language characteristics. To date, the comparison between classroom-based and environment-based multilinguals is available only across studies and paradigms. Studies that directly compare such populations to isolate the effects of speakers' prior learning circumstances should be conducted. Similarly, studies isolating the role of other learner characteristics (proficiency and AoA), language similarity and the number of known languages, are needed. Moreover, the reviewed literature points to interesting differences between children and adults in the weight of direct versus indirect effects of multilingualism, with stable direct effects across the life span and more isolated documentation of indirect influences across age. Within-study comparisons across age groups would serve to enhance our understanding of such differences.

As noted in Fig. 1, several additional aspects are not represented in the literature. From the perspective of the learner, we know little on how the circumstances of language use (including the speakers' switching habits) and individual differences in the baseline level of cognitive and social abilities might affect the interplay of direct and indirect influences. From the perspective of the to-be-learned language, as mentioned earlier, the literature is heavily skewed toward vocabulary learning with very little work on grammar and literacy. Moreover, how multilingualism influences learning of the target language's accent is unknown. Similarly, if and how multilingualism influences the attainment of fluency in the novel language, above and beyond initial learning, remains to be examined.

Finally, the current review focused on uncovering the cognitive underpinnings of a possible multilingualism advantage 
in language learning. The reflection of these mechanisms in brain structure and function remains to be discovered.

\section{Conclusion}

The present article presents a comprehensive review of the effects of multilingualism on language learning, focusing on learning of novel vocabulary, phonology, grammar, and literacy. We propose a framework by which the effect of multilingualism could operate through a direct pathway by which prior experiences (including knowledge and skills) are being transferred to novel learning situations as a function of the similarity of the novel material and situation to prior experiences. At the same time, multilingualism may affect language learning by way of an indirect pathway, operating through general cognitive and linguistic abilities, which result from continuous use of more than one language. We further note that these direct and indirect effects are partially modulated by the characteristics of the learner and of the languages in question. The literature shows that the multilingual advantage is present for both children and adults and that both direct and indirect mechanisms operate across the life span. Similarly, prior language learning context (classroom based vs. environment based) appears to influence the balance between direct and indirect effects, such that in formal language learning settings, classroom-based multilinguals exhibit direct effects whereas environment-based multilinguals rely more on indirect effects. Finally, the literature on the effects of multilingualism on grammar and literacy, and in particular studies which uncover the underlying mechanisms by which multilingualism may affect language learning in these domains, is scarce. To conclude, the degree to which multilingualism affects novel language learning provides evidence that relates to the dynamic nature of the linguistic and cognitive system and its developmental course, and serves to complement the literature on the effect of multilingualism on language processing and cognition more broadly.

Acknowledgements This work was supported by an EU-FP7 Grant CIG-322016 and the Language Learning Small Grants Research Program. The authors wish to thank Anat Prior, Jared Linck, and an anonymous reviewer for their insightful comments on an earlier version of the manuscript.

\section{References}

Abu-Rabia, S., \& Sanitsky, E. (2010). Advantages of bilinguals over monolinguals in learning a third language. Bilingual Research Journal, 33, 173-199.

Antoniou, M., Liang, E., Ettlinger, M., \& Wong, P. (2015). The bilingual advantage in phonetic learning. Bilingualism: Language and Cognition, 18, 283-295.
Au, T. K., \& Glusman, M. (1990). The principle of mutual exclusivity in word learning: To honor or not to honor? Child Development, 61, 1474-1490.

Bartolotti, J., \& Marian, V. (2012). Language learning and control in monolinguals and bilinguals. Cognitive Science, 36, 1129-1147.

Bartolotti, J., Marian, V., Schroeder, S. R., \& Shook, A. (2011). Bilingualism and inhibitory control influence statistical learning of novel word forms. Frontiers in Psychology, 2, 324.

Bialystok, E., Kroll, J. F., Green, D. W., MacWhinney, B., \& Craik, F. I. M. (2015). Publication bias and the validity of evidence: What's the connection? Psychological Science, 26, 944-946.

Bialystok, E., Luk, G., \& Kwan, E. (2005). Bilingualism, biliteracy, and learning to read: Interactions among languages and writing systems. Scientific Studies of Reading, 9, 43-61.

Bjork, R. A., \& Kroll, J. F. (2015). Desirable difficulties in vocabulary learning. The American Journal of Psychology, 128, 241-252.

Blumenfeld, H. K., \& Adams, A. M. (2014). Learning and processing of nonverbal symbolic information in bilinguals and monolinguals. Frontiers in Psychology, 5, 1147.

Brojde, C. L., Ahmed, S., \& Colunga, E. (2012). Bilingual and monolingual children attend to different cues when learning new words. Frontiers in Psychology, 3, 155.

Byers-Heinlein, K., \& Werker, J. F. (2009). Monolingual, bilingual, trilingual: Infants' language experience influences the development of a word-learning heuristic. Developmental Science, 12, 815-823.

Byers-Heinlein, K., \& Werker, J. F. (2013). Lexicon structure and the disambiguation of novel words: Evidence from bilingual infants. Cognition, 128, 407-416.

Davis, C., \& Bryant, P. (2006). Causal connections in the acquisition of an orthographic rule: A test of Uta Frith's developmental hypothesis. Journal of Child Psychology and Psychiatry, 47, 849-856.

De Groot, A. M. B. (1992). Determinants of word translation. Journal of Experimental Psychology: Learning, Memory, and Cognition, 18, 1001-1018.

Degani, T., \& Tokowicz, N. (2010). Semantic ambiguity within and across languages: An integrative review. The Quarterly Journal of Experimental Psychology, 63, 1266-1303.

Errasti, M. P. S. (2003). Acquiring writing skills in a third language: The positive effects of bilingualism. International Journal of Bilingualism, 7, 27-42.

Frost, R., Armstrong, B. C., Siegelman, N., \& Christiansen, M. H. (2015). Domain generality versus modality specificity: The paradox of statistical learning. Trends in Cognitive Sciences, 19, 117-125.

Gollan, T. H., Montoya, R. I., Cera, C., \& Sandoval, T. C. (2008). More use almost always means a smaller frequency effect: Aging, bilingualism, and the weaker links hypothesis. Journal of Memory and Language, 58, 787-814.

Halberda, J. (2003). The development of a word-learning strategy. Cognition, 87, B23-B34.

Herdina, P., \& Jessner, U. (2002). A dynamic model of multilingualism: Perspectives of change in psycholinguistics. Clevedon: Multilingual Matters.

Ivanova, I., \& Costa, A. (2008). Does bilingualism hamper lexical access in speech production? Acta Psychologica, 127, 277-288.

Jessner, U. (2008). A DST Model of multilingualism and the role of metalinguistic awareness. The Modern Language Journal, 92, 270-283.

Kahn-Horwitz, J., Kuash, S., Ibrahim, R., \& Schwartz, M. (2014). How do previously acquired languages affect acquisition of English as a foreign language: The case of Circassian. Written Language \& Literacy, 17, 40-61.

Kahn-Horwitz, J., Schwartz, M., \& Share, D. (2011). Acquiring the complex English orthography: A triliteracy advantage? Journal of Research in Reading, 34, 136-156.

Kalashnikova, M., Mattock, K., \& Monaghan, P. (2014). The effects of linguistic experience on the flexible use of mutual exclusivity in 
word learning. Bilingualism: Language and Cognition, 18, 626638 .

Kaushanskaya, M. (2012). Cognitive mechanisms of word learning in bilingual and monolingual adults: The role of phonological memory. Bilingualism: Language and Cognition, 15, 470-489.

Kaushanskaya, M., Gross, M., \& Buac, M. (2014). Effects of classroom bilingualism on task-shifting, verbal memory, and word learning in children. Developmental Science, 17, 564-583.

Kaushanskaya, M., \& Marian, V. (2009a). The bilingual advantage in novel word learning. Psychonomic Bulletin \& Review, 16, 705-710.

Kaushanskaya, M., \& Marian, V. (2009b). Bilingualism reduces nativelanguage interference during novel-word learning. Journal of Experimental Psychology. Learning, Memory, and Cognition, 35, 829-835.

Kaushanskaya, M., \& Prior, A. (2015). Variability in the effects of bilingualism on cognition: It is not just about cognition, it is also about bilingualism. Bilingualism: Language and Cognition, 18, 27-28.

Kaushanskaya, M., \& Rechtzigel, K. (2012). Concreteness effects in bilingual and monolingual word learning. Psychonomic Bulletin \& Review, 19, 935-941.

Kaushanskaya, M., Yoo, J., \& Van Hecke, S. (2013). Word learning in adults with second language experience: Effects of phonological and referent familiarity. Journal of Speech, Language, and Hearing Research, 56, 667-678.

Kemp, C. (2007). Strategic processing in grammar learning: Do multilinguals use more strategies? International Journal of Multilingualism, 4, 241-261.

Keshavarz, M. H., \& Astaneh, H. (2004). The impact of bilinguality on the learning of English vocabulary as a foreign language (L3). International Journal of Bilingual Education and Bilingualism, 7, 295-302.

Klein, E. C. (1995). Second versus third language acquisition: Is there a difference ? Language Learning, 45, 419-465.

Kroll, J. F., \& Bialystok, E. (2013). Understanding the consequences of bilingualism for language processing and cognition. Journal of Cognitive Psychology, 25, 497-514.

Linck, J. A., Hoshino, N., \& Kroll, J. F. K. (2008). Cross-language lexical processes and inhibitory control. Mental Lexicon, 3, 349-374.

Linck, J., Michael, E., Golonka, E., Twist, A., \& Schwieter, J. W. (2015). Moving beyond two languages: The effects of multilingualism on language processing and language learning. In J. W. Schwieter (Ed.), The Cambridge handbook of bilingual processing. Cambridge: Cambridge University Press.

Mady, C. (2014). Learning French as a second official language in Canada: Comparing monolingual and bilingual students at Grade 6. International Journal of Bilingual Education and Bilingualism, $1,330-344$

Markman, E. M. (1990). Constraints children place on word meanings. Cognitive Science, 14, 57-77.

Markman, E. M., Wasow, J. L., \& Hansen, M. B. (2003). Use of the mutual exclusivity assumption by young word learners. Cognitive Psychology, 47, 241-275.

Modirkhamene, S. (2006). The reading achievement of third language versus second language learners of English in relation to the interdependence hypothesis. International Journal of Multilingualism, 3, 280-295.

Paap, K. R., Johnson, H. A., \& Sawi, O. (2015). Bilingual advantages in executive functioning either do not exist or are restricted to very specific and undetermined circumstances. Cortex, 69, 265-278.
Papagano, C., \& Vallar, G. (1995). Verbal short-term memory and vocabulary learning in polyglots. The Quarterly Journal of Experimental Psychology Section A, 48, 98-107.

Sanz, C. (2000). Bilingual education enhances third language acquisition: Evidence from Catalonia. Applied Psycholinguistics, 21, 23-44.

Sanz, C. (2007). Predicting enhanced L3 learning in bilingual contexts: The role of biliteracy. In C. Pérez-Vidal, M. Juan-Garau, \& A. Bel (Eds.), A portrait of the young in the new multilingual Spain (pp. 220-240). Buffalo: Multilingual Matters.

Schwartz, M., Geva, E., Share, D. L., \& Leikin, M. (2007). Learning to read in English as third language: The cross-linguistic transfer of phonological processing skills. Written Language and Literacy, 10, $25-52$.

Schwartz, M., Kahn-Horwitz, J., \& Share, D. L. (2014). Orthographic learning and self-teaching in a bilingual and biliterate context. Journal of Experimental Child Psychology, 117, 45-58.

Siegelman, N., Bogaerts, L., Chrisiansen, M. H., \& Frost, R. (2016). Towards a theory of individual differences in statistical learning. Philosophical Transactions of the Royal Society B, 372(1711). doi: 10.1098/rstb.2016.0059

Siegelman, N., \& Frost, R. (2015). Statistical learning as an individual ability: Theoretical perspectives and empirical evidence. Journal of Memory and Language, 81, 105-120.

Sommers, M. S., \& Barcroft, J. (2013). Effects of referent token variability on L2 vocabulary learning. Language Learning, 63, 186-210.

Stafford, C. A., Sanz, C., \& Bowden, H. W. (2010). An experimental study of early L3 development: Age, bilingualism and classroom exposure. International Journal of Multilingualism, 7, 162-183.

Tokowicz, N., \& Degani, T. (2015). Learning second language vocabulary: Insights from laboratory studies. In J. W. Schwieter (Ed.), The Cambridge handbook of bilingual processing. Cambridge: Cambridge University Press.

Trembley, M. C., \& Sabourin, L. (2012). Comparing behavioral discrimination and learning abilities in monolinguals, bilinguals and multilinguals. The Journal of the Acoustical Society of America, 132, 3465-3474.

Valian, V. (2015). Bilingualism and cognition. Bilingualism: Language and Cognition, 18, 3-24.

Van Assche, E., Duyck, W., \& Gollan, T. H. (2013). Whole-language and item-specific control in bilingual language production. Journal of Experimental Psychology: Learning, Memory, and Cognition, 39, 1781.

Van Gelderen, A., Schoonen, R., De Glopper, K., Hulstijn, J., Snellings, P., Simis, A., \& Stevenson, M. (2003). Roles of linguistic knowledge, metacognitive knowledge and processing speed in L3, L2 and L1 reading comprehension: A structural equation modeling approach. International Journal of Bilingualism, 7, 7-25.

Van Hell, J. G., \& Candia Mahn, A. (1997). Keyword mnemonics versus rote rehearsal in learning concrete and abstract foreign words by experienced and inexperienced foreign language learners. Language Learning, 47, 507-546.

Van Hell, J. G., \& De Groot, A. M. B. (1998). Conceptual representation in bilingual memory: Effects of concreteness and cognate status in word association. Bilingualism: Language and Cognition, 1, 193211.

Wang, T., \& Saffran, J. R. (2014). Statistical learning of a tonal language: The influence of bilingualism and previous linguistic experience. Frontiers in Psychology, 5, 953.

Yoshida, H., Tran, D. N., Benitez, V., \& Kuwabara, M. (2011). Inhibition and adjective learning in bilingual and monolingual children. Frontiers in Psychology, 2, 210. 